\title{
MEMBRANE-TRANSFERRING REGIONS OF GP41 AS TARGETS FOR HIV-1 FUSION INHIBITION AND VIRAL NEUTRALIZATION.
}

Nerea Huarte ${ }^{1}$, Maier Lorizate ${ }^{2}$, Enrique Pérez-Payá ${ }^{3,4}$, and José L. Nieva*

${ }^{1}$ Unidad de Biofísica (CSIC-UPV/EHU) and Dpt. de Bioquímica y Biología Molecular, Universidad del País Vasco, Aptdo. 644, E-48080 Bilbao, Spain.

${ }^{2}$ Department of Infectious Diseases, Virology, University Hospital of Heidelberg, Heidelberg D-69120, Germany

${ }^{3}$ Dpt. Quimica Medica. Centro de Investigacion Principe Felipe. E-46013 Valencia, Spain, ${ }^{4}$ Instituto de Biomedicina de Valencia (CSIC). E-46010 Valencia, Spain.

*Corresponding author

Abstract: The fusogenic function of HIV-1 gp41 transmembrane Env subunit relies on two different kinds of structural elements: i) a collapsible ectodomain structure (the hairpin or six-helix bundle) that opens and closes, and ii) two membrane-transferring regions (MTRs), the fusion peptide (FP) and the membrane-proximal external region (MPER), which ensure coupling of hairpin closure to apposition and fusion of cell and viral membranes. The isolation of naturally produced short peptides and neutralizing IgG-s, that interact with FP and MPER, respectively, and block viral infection, suggests that these conserved regions might represent useful targets for clinical intervention. Furthermore, MTR-derived peptides have been shown to be membrane-active. Here, it is discussed the potential use of these molecules and how the analysis of their membrane activity in vitro could contribute to the development of HIV fusion inhibitors and effective immunogens. 


\section{Introduction: membrane-transferring regions within the globular gp41 ectodomain.}

Membrane fusion comprises a multi-step, highly regulated physiological reaction that proceeds mechanically coupled to the basic processes of local rupture (exposure of the hydrophobic interior) and deformation (bending) of lipid bilayers (reviewed in [1-4]). The human immunodeficiency virus type-1 (HIV-1) relies on the fusogenic activity of the envelope glycoprotein (Env) to enter and infect its $\mathrm{CD}^{+}{ }^{+}$host cells [5-7]. The HIV-1 fusion machinery shares a number of common features with other fusogenic viral glycoproteins [8-11]: functional priming at the cell surface through proteolytic processing of Env gp160 precursor to render non-covalently bound surface (gp120) and transmembrane (gp41) subunits; an oligomeric organization (homotrimers of gp120gp41 heterodimers); fusogenic activity localized into ecto- and transmembrane domains of the integral gp41 subunit; and the presence of a fusion peptide (FP), a highly conserved, hydrophobic domain, which, in the case of HIV-1, is located at the free amino terminus of the fusogenic gp41 subunit.

From a structural point of view, HIV-1 Env belongs to the "Class I" fusion proteins, characterized by their transmembrane subunit's capacity to fold into a highly stable 6helix bundle $(6-\mathrm{HB})[6,10,11]$. In this "hairpin-like" trimeric structure three helices pack in the reverse direction against hydrophobic grooves outside a triple-stranded coiled-coil, so that the ectodomain amino- and carboxy-termini are placed at the same end of the molecule (Figure 1). It is generally assumed that amino-terminal FP sequences insert into the target cell membrane in the "pre-hairpin" stage created upon receptor/co-receptor binding [12-14]. Subsequent production of the 6-HB structure 
would therefore induce close apposition of the viral and cell membranes anchored through the transmembrane domain (TMD) and the FP, respectively. According to this view, the N-terminal FP would function as an inert anchor, i.e., a "secondary" TMD that, together with the "primary" TMD, would transmit the force generated by 6-HB formation into the target membrane.

However, two main observations point to an active role of gp41 ectodomain sequences at modulating the bilayer elastic stress during membrane fusion. In one hand, mutant fusion domains have been isolated that are capable of integrating into target bilayers, and yet they do not support fusion activity $[15,16]$ indicating that simple insertion as an inert membrane anchor is not enough to unleash FP function. On the other hand, in the context of the 6-HB, the C-terminal helices are not directly followed by TMDs (Figure 1A). In HIV-1 an intervening sequence of approximately 20 amino acids, termed as the membrane proximal external region (MPER), separates both gp41 domains $[17,18]$. In fact, a soluble and structurally irregular MPER linker might keep the 6-HB far from the close contacting dehydrated areas required for membrane fusion initiation (as far as 70 $\AA$ apart from the viral membrane). It was thus argued that the transmission of the force generated upon folding into the 6-HB required the establishment of direct contacts between the MPER and the HIV membrane [19].

Wimley-White (WW) hydrophobicity scales can provide valuable tools for the detection of membrane-active domains, particularly useful in the case of viral products that insert into membranes of the host cells (reviewed in [20, 21]). These scales are based on partitioning free energies of individual amino acids (including the peptide bond) from water into membrane interfaces $\left(\Delta \mathrm{G}_{\text {wiu }}\right)$ or octanol $\left(\Delta \mathrm{G}_{\text {wio }}\right)[22]$. When applied to HIV-1 gp41 ectodomain the hydrophobicity-at-interface WW scale displays a positive peak that is indeed coincident with the N-terminal FP sequence (Figure 1A). In addition, a 
positive maximum spanning MPER is detected at the $\mathrm{C}$-terminus, thereby indicating that both hairpin ends are endowed with the capacity for transferring from water into the membrane interface (MI). The gp41 ectodomain membrane-transferring regions (MTRs) can be operationally defined based on two distinct characteristics: i) their favorable free energy of partitioning from water into the MI; and ii) their intrinsic capacity for perturbing the lipid bilayer architecture upon insertion (membrane activity).

Thus, MTR refer to sequences, other than the TMD, which bear the potential for partitioning from water into the membrane interface [20], and that by doing so may compromise the bilayer integrity [18]. However, these sequences do not function as membrane anchors recognized by the translocon as stably integrated transmembrane helices during glycoprotein synthesis [23]. Explicitly, in the full gp41 sequence, the ectodomain FP and MPER sequences [24-26], and the cytoplasmic tail lentivirus lytic peptides (LLPs) [24, 27, 28], would fulfill the requirements to qualify as MTRs.

MTR partitioning into membranes has been quantitatively assessed in vesicular model systems using representative synthetic peptides [12, 29-37]. These studies not only confirm the predicted high affinity of MTRs for the membrane interface, but also support their role as promoters of lipid bilayer destabilization [12, 13, 34, 36-38]. In principle MTR-s might promote fusion by reducing the formation energies of the lipidic intermediates of fusion, and/or by accelerating the opening of fusion pores [39-41]. Given their common membrane topology at equilibrium, i.e., inserted at the level of the interface-hydrocarbon region of one monolayer, it has been proposed that MTRs may induce lateral stress in the hydrocarbon region of the external monolayer and/or selectively induce an increment of its surface [34, 42-45]. These effects would result in the creation of bulges (or dimples) that protrude from the membrane plane [46]. The tops of these structures are highly curved, an arrangement that can facilitate the 
formation of lipidic contacts between fusing bilayers [46]. Alternatively, based on the evidence that MTRs may lower the membrane rupture tension [47], it has been proposed that a plausible mechanism of MTR-mediated fusion-promotion could be the destabilization of bilayer diaphragms at hemifusion intermediates [40]. Yet another line of evidence derived from diffuse x-ray scattering experiments, indicates that the FP inserted in membranes might reduce the bending energy, and, hence, facilitate the formation of curved lipidic intermediates of fusion [41].

\section{II: HIV-1 fusion inhibition by targeting the fusion peptide}

The most widely accepted mechanism of HIV-1 Env-mediated fusion introduced in the previous section encompasses two key processes (Figure 1B): 1) Insertion of MTRs into the fusing membranes, and 2) Collapse of the pre-hairpin intermediate into an energetically stable 6-HB structure. Since entry inhibition may block early stages of viral infection, the large conformational changes that are the energy source for membrane fusion constitute an important target in development of antiviral therapies. The HIV fusion inhibitors derived from the C-terminal heptad repeat region (CHR) comprise a family of peptide-based compounds, some of them, such as the T-20 derivatives, currently in clinical use [48]. These molecules dominantly compete with gp41 helical sequences for binding to hydrophobic grooves transiently exposed at the ectodomain surface $[5,6]$. Thus, it is postulated that T-20-like peptides block close contact between cell target and viral membranes by preventing 6-HB formation. Although highly effective, the clinical use of these compounds is circumscribed to 
rescue therapies against multidrug resistant viruses [48]. Bioavailability issues, including rapid proteolytic degradation, and consequent requirement of high doses delivered by injection, pose a serious limitation to their wider application.

In theory the gp41 MTRs that transfer into and perturb membranes may comprise alternative/complementary targets for antiviral development. MTR membrane-activity is dependent on the adoption of structurally defined oligomeric complexes at membrane surfaces, a requirement that might explain, at least in part, their high degree of sequence conservation [19, 30-32]. Mutagenesis studies have put forward the absolute requirement of the FP sequence for gp41 fusogenic activity [49-51]. Amino acid homology between fusion proteins of different viruses is usually of less than $20 \%$. In contrast FP homology for the same proteins can be as high as 90\% [52]. FPs are also unusually enriched in Ala and Gly residues [53]. Comparable high degrees of residue conservation and Ala/Gly content are not found in other membrane-associated hydrophobic protein domains as signal sequences or transmembrane anchors, suggesting that FP sequences have evolved constrained by a selective pressure that cannot be explained by simple requirements of hydrophobicity or amphipathicity. Supporting the existence of a tight structure-function relationship, Longo et al. [54] identified a pore-formation activity of the functional FP well below the membrane rupture tensions caused by simple transfer of mass to the bilayers. Thus, the ability to perturb the bilayer architecture after insertion seems to be an intrinsic feature of functional viral FPs, which probably requires formation of specific membraneembedded complexes [42].

Interference with formation of these structurally defined oligomeric complexes might also explain the inhibitory effect displayed by short peptides derived from the FP sequence in cell systems. A hexapeptide which was identical in amino acid sequence to 
the gp41 N-terminus was found by Owens and coworkers [55] to completely inhibit syncitium formation mediated by HIV-1 envelope glycoproteins expressed at the surface of HeLa CD4+ cells. The inhibitory effect was sequence-specific and dosedependent. Longer HIV-1 FPs inhibited HIV-induced syncytium formation and antigen production in infected cells [56]. When peptide solubility was increased by a Cterminus conjugation to charged polymers an increase in inhibitory activity was found; in contrast, such modification to the N-terminus abolished inhibition. Kliger and coworkers [31] demonstrated inhibition of cell-cell fusion induced by expressed gp120/41 using 33-mer sequences representing the gp41 N-terminus. Again a functional feature of these sequences was shown to be their capacity to assemble high order complexes. In later developments, these authors demonstrated that both, a synthetic all D-amino acid peptide (D-amino acid stands for the use of the D-stereoisomers of the natural amino acids) corresponding to the same sequence, and a peptide in which the highly conserved Ile4, Phe8, Phe11, and Ala14 residues were replaced by their Dcounterparts, were able to bind to the wild-type peptide in the membrane, inhibiting cell fusion mediated by the HIV-1 envelope glycoprotein [57, 58].

Thus, FP function emerges as an alternative therapeutic target for the development of fusion inhibitors. Supporting this view, Münch and co-workers [59] have recently reported the isolation of a naturally produced blood plasma oligopeptide, capable of blocking HIV-1 infection by targeting gp41 FP. This peptide, termed by these authors as VIRIP (virus-inhibitory peptide), was purified from hemofiltrate obtained from patients suffering from chronic renal failure. VIRIP was identified as a derivative from the Cterminal sequence of circulating serine protease inhibitor $\alpha 1$-antitrypsin. This original sequence inhibited infection of PBMC by HIV-1 with $\mathrm{IC}_{50}$-s of about $20 \mu \mathrm{M}$, in a highly sequence-specific manner. Even though several residues proved to be absolutely 
required for the inhibitory activity, VIRIP could be optimized by adding non-natural amino acids at positions that tolerated substitution by Ala, and/or by introducing intramolecular cyclic motifs by disulfide bonds. This optimized versions resulted as potent as T-20 in blocking viral infection and were devoid of cellular toxicity.

The FP sequence was identified as the VIRIP's molecular target as evidenced by its capacity for inhibiting synthetic FP-promoted hemolysis [60]. Notably, a positive correlation was established between anti-hemolytic activity and infection inhibition capacity, i.e., VIRIP derivatives showing improved anti-HIV capacity were also more potent interfering with FP-induced hemolysis. Finally, the structure of FP in complex with the optimized VIRIP derivative VIR-165, was elucidated in NMR experiments (PDB entry: 2JNR). Structural analyses of the complex revealed that these peptides bracket together via hydrophobic interactions of the amino acid side chains.

More recently, Forsmann et al. [61] have reported on the outcome of a 10-day monotherapy with the optimized VIR-576 derivative of VIRIP applied to HIV-1infected patients. These authors evaluated the safety, pharmacokinetics and antiviral efficacy of VIR-576 in 18 patients. Treatment with VIR-576 was well tolerated by patients, and reduced their plasma viral load by $1.23 \log _{10}$ copies. These promising results constitute a first proof of concept that VIRIP can suppress viral replication in infected individuals, and support the clinical potential of FP inhibitors.

\section{Synthetic peptide combinatorial libraries as a potential source of antiFP compounds.}

Eckert et al. [62] described inhibitory peptides based on D-amino acids that targeted a 
pocket on the surface of the central trimeric coiled-coil within gp41. These D-amino acid-based pocket-specific inhibitors of entry (PIE) have been more recently optimized by Welch et al. [63]. As discussed by these authors, D-amino acid-based peptides with fusion inhibitory activities have several potential advantages over the natural L-amino acid-based peptide inhibitors: i) they are resistant to proteases; ii) they have the potential to be administered orally; and iii) they are structurally more diverse, a feature allowing easier targeting to geometrically complex surfaces.

Synthetic combinatorial peptide libraries have been also developed based on D-amino acids. In particular, Houghten's laboratory developed in the nineties the concept of positional scanning synthetic combinatorial libraries (PS-SCL; $[64,65]$ ) that was also later explored by others [66-68]. In the peptide-based PS-SCL the peptide mixtures used to be $\mathrm{C}$-amidated and $\mathrm{N}$-acetylated. The most popular size for these libraries is the hexapeptide. Then an hexapeptide-D-amino acid-based library is composed of six individual positional SCLs represented by the formulae: Ac- $0_{1} x x x x x-N H 2$, Ac$\mathrm{xo}_{2} \mathrm{xxxx}-\mathrm{NH} 2, \quad \mathrm{Ac}-\mathrm{xxO}_{3} \mathrm{xxx}-\mathrm{NH} 2, \mathrm{Ac}-\mathrm{xxxO}_{4} \mathrm{xx}-\mathrm{NH} 2, \mathrm{Ac}-\mathrm{xxxxO}_{5} \mathrm{x}-\mathrm{NH} 2$ and Ac$\mathrm{xxxxxo}_{6}-\mathrm{NH} 2$ (o: defined by one of 20 D-amino acids; $\mathrm{x}$ : close to equimolar mixture of 19 D-amino acids, cysteine omitted). Each peptide mixture is composed of approximately 2.5 million $\left(19^{5}\right)$ individual hexapeptides, for a total of over 50 million $\left(20 \times 19^{5}\right)$ hexamers. Each positional SCL addresses a separate single position of the sequence, and the PS-SCL approach relies on the connectivity between the amino acids defining the most biologically active mixtures at each positions being addressed. It was early demonstrated that the screening of a D-amino acid-based PS-SCL was useful for the identification of inhibitors of membrane active natural (i.e., L-amino acid) peptides such as melittin through peptide-peptide interactions $[69,70]$. 


\section{Part IV. antiFP isolation from a PS-SCL based on pore-forming membrane activity}

In search for alternative fusion inhibitors we screened a PS-SCL for the presence of anti-MTR compounds. Analysis of the molecular mechanisms underlying MTR activity in the whole gp41 protein is not viable as yet. This has warranted the development of in vitro assays based on the use of representative synthetic peptides and model membranes $[21,34]$. The ANTS/DPX leakage assay for pore formation is widely used to assess the membrane activity of bioactive peptides in liposomes [71]. In particular, using this assay a significant correlation was established between the effects of FP mutations blocking gp41 fusogenic activity, and the effects displayed by the same sequence alterations on the capacity of synthetic analogs to form lytic pores in vesicles $[29,30$, 32, 33, 42]. The process of FP pore formation in 1-palmitoyl-2oleoylphosphatidylglycerol (POPG) vesicles has been successfully modeled by Nir and co-workers $[29,72]$ under the following mechanistic assumptions (Figure 2A):

1) First a rapid stage of peptide binding to the vesicle membranes takes place (see also panel C).

2) Coupled to partitioning, the FP attains a helical structure at the membraneinterface.

3) Following the structuring step, a surface aggregation of helical peptides occurs.

4) When the structure and conformation of the peptides are appropriate, the aggregates that have reached a critical size will form a permeating pore.

In this model, the kinetics of leakage (panel C) is dictated by the kinetics of surface 
aggregation, bilayer translocation and aqueous pore opening, since once a pore has formed the leakage of all the contents occurs within less than $1 \mathrm{~s}$, thus yielding a finite final extent. In accordance with this model, FP blocking agents may potentially act by interfering with partitioning (i.e., generating a water soluble complex with reduced $\Delta \mathrm{G}_{\mathrm{wiu}}$ ), hindering the conformational changes and/or oligomerization processes taking place at the bilayer surface, or occluding the aqueous channel.

FP-dependent pore formation in POPG vesicles was therefore used as the functional assay to screen the PS-SCL for the identification of compounds that inhibited FP activity [73]. The peptide mixtures that defined the library, were pre-mixed with FP in DMSO prior to addition to the POPG large unilamellar vesicle (LUV) suspension. Figure 2B illustrates the effects on FP-induced leakage induced by representative mixtures in the Ac- $\mathrm{xxO}_{3} \mathrm{Xxx}-\mathrm{NH} 2$ sub-library (defined amino acids at position 3). Each sub-library addressed a separate position of the hexapeptide and, when used in concert, the data provided information about the most important amino acid residue at each position [74]. The so-called "library deconvolution" consisted in selecting the most active mixtures as inhibitors, and identifying the relevant amino acid residues for each position. The screening data allowed not only the selection of amino acids at each position, which should lead to active individual sequences, but also gave information on the relative importance of each residue within the active sequences. Our criteria for the selection of amino acids for the synthesis of individual sequences derived from the PSSCL were significantly higher inhibitory activity as compared to the peptide mixtures within the same single positional SCL, and/or difference in chemical character within the same single positional SCL and/or throughout the PS-SCL. As a result of this focused process the following mixtures were selected: $g(1)-p / q / s(2)-i(3)-d(4)-e / q(5)-$ $\mathrm{v}(6)$. Then $1 \times 3 \times 1 \times 1 \times 2 \times 1=6 \mathrm{D}$-amino acid hexapeptide sequences were synthesized and 
assayed against FP. From this initial set, three sequences resulted clearly inhibitory (ie., they induced $>50 \%$ reduction in observed leakage): Ac-gqidev-NH2, Ac-gqidqv-NH2 and Ac-gsidqv-NH2. Moreover, among these sequences, Ac-gqidev-NH2 (82-2) and Ac-gqidqv-NH2 (82-5) also inhibited syncytium formation induced by gp41 expressed at cell surfaces.

Preliminary data displayed in Figures 2 C,D advance some clues on the mechanism underlying FP-induced leakage inhibition by the isolated 82-2 and 82-5 hexapeptides. Insertion into the non-polar lipid bilayer milieu of FP fluorescently labeled with NBD caused a sudden increase in the fluorescence emission intensity of the probe (dotted line in panel C). Inhibitory D-hexapeptides appear to not interfere with water-membrane partitioning as judged from their incapacity to preclude this insertion process (not shown). The NBD fluorescence increase, which was not blocked by hexapeptide addition, was followed by the onset of ANTS/DPX leakage. In contrast to insertion, Dhexapeptide addition blocked ongoing leakage from vesicles that had incorporated the FP, suggesting that the membrane-bound FP population may constitute the actual target for the inhibitors.

As shown in Figure 2E, the 82-2 and 82-5 compounds inhibited fusion in a dosedependent manner showing apparent $50 \%$ inhibitory concentration $\left(\mathrm{IC}_{50}\right)$ values of ca. $20 \mu \mathrm{M}$ and $100 \mu \mathrm{M}$, respectively. The observation that a sequence bearing D-proline in position 2 (Ac-gpidqv-NH2) was not inhibitory of FP membrane activity or gp41induced cell-cell fusion emphasizes the specificity of the process. The inhibitory potency of these compounds was ca. x500-1000 times lower (when weight was considered) than that exhibited by the T-20 peptide in the same assay [73], but nonetheless comparable to that reported for the first generation of PIEs in similar cellcell fusion inhibition assays [62]. Research work is under progress in our laboratories in 
order to precisely elucidate the stage between bilayer partitioning and pore-opening that is targeted by D-hexapeptides. Our expectation is that characterizing the inhibition mechanism of pore-formation in the vesicular systems will provide valuable starting information to attempt the resolution of the mechanism underlying gp41-induced fusion inhibition. We also surmise that isolating the FP stage relevant for inhibition may guide future structural studies. Recent structure-assisted redesign of PIE sequences has yielded optimized versions displaying $\mathrm{IC}_{50}$ values in the order of $\mathrm{pM}$ in neutralization assays [63]. Thus, the first generation of anti-FP D-hexapeptides might also comprise leading platforms for inhibitor optimization in the near future.

\section{MPER structure-function}

The MPER sequence encompasses the second class of gp41 MTR. Based on mean interfacial hydrophobicity calculations we proposed this sequence to span ${ }^{664}$ DKWASLWNWFNITNWLWYIK ${ }^{683}$ Env residues $[18,24]$. Compelling mutational analysis by Salzwedel and co-workers [17] provided evidence to support the involvement of this interfacial gp41 stretch in HIV-1 fusion. Deletion of the entire sequence (Env residues 665-682) abrogated the ability of the glycoprotein to mediate

cell-cell fusion without affecting the normal maturation, transport, or CD4-binding ability of the protein. The same fusion phenotype was demonstrated for W(1-5)A, an MPER mutant devoid of interfacial hydrophobicity by substituting alanine residues for 
the five tryptophan residues within this sequence $[17,21]$. Subsequent functional studies revealed that membrane fusion induced by gp41 devoid of MPER interfacial hydrophobicity was arrested at the stage of non-expanding small fusion pores [75], which suggests a role during post-lipid mixing stages [76].

The membrane-activity of MPER-derived peptides detected in vitro provides experimental evidence to support a functional role for this gp41 MTR during fusion [18, 25, 77]. Characterization of the HIV-1 lipidome has revealed high levels of cholesterol (Chol) and sphingomyelin (SPM) [78-80], an observation that correlates with the selectivity for specific segregated membrane regions through which virions emerge during maturation $[81,82]$. The membrane activity of the MPER-derived peptide ${ }^{664}$ DKWASLWNWFNITNWLWYIK ${ }^{683}$ (PreTM) was modulated by these envelope lipids [77]. Specifically, Chol greatly stimulated PreTM's lytic activity and activated its fusogenic capacity [77]. When PC, SPM and Chol were combined to emulate conditions of liquid ordered (Lo)-liquid disordered (Ld) lipid domain segregation, putatively existing at the external membrane monolayer of HI virion, high levels of PreTM surface self-aggregation, concomitant to an enhancement in fusion and lytic activities were observed at low peptide doses (in the order of 1:1000 peptide-to-lipid ratio). Conditions described to disturb occurrence of lateral separation of Lo-Ld lipid phases in these systems, reproduced the high peptide-dose requirements for leakage as found in pure PC vesicles and inhibited fusion [77]. Thus, the lipid dependence observed in the vesicular systems suggests a functional role for MPER membrane activity in destabilizing the envelope lipid bilayer architecture at the loci of fusion.

The possible existence of a direct interaction between Chol and PreTM domain was already posited by Salzwedel et al.[17]. MPER carboxy-terminal LWYIK sequence has been identified as a potential "cholesterol recognition/interaction amino acid consensus" 
(CRAC) domain functional in fusion [83-87]. Recently reported experimental evidence and modeling studies provide the physicochemical grounds for a direct interaction of MPER-CRAC with Chol [84, 85, 88, 89]. MPER-Chol interaction would be based on two capacities of CRAC sequence, namely, wrapping and blocking of the interfacial cholesterol $\mathrm{OH}$ group by $\mathrm{H}$-bond interactions, and stacking of aromatic side chains with A ring of cholesterol.

The functional importance of MPER lytic activity for gp41-induced fusion has been recently demonstrated by Vishwanathan and Hunter [90]. These authors designed gp41 chimeras that replaced all or part of MPER with indolicidin-based sequences. Indolicidin is a Trp-rich, antimicrobial and hemolytic peptide that forms pores in membranes. Thus, indolicidin and MPER have in common the unusual high number of tryptophans and their capacity to permeabilize membranes. These authors hypothesized that if MPER-induced membrane perturbations were required for gp41 fusogenic function, its replacement with indolicidin would produce functional Env glycoprotein. Some of the produced gp41 chimeras indeed retained fusion activity [90], therefore demonstrating that MPER can be replaced by a membrane-disruptive foreign sequence and preserve its biological function.

Structural studies support that membrane-active MPER may fold within the lowpolarity membrane-interface environment adopting a helical conformation, and also self-aggregate therein (Figure 3). NMR spectroscopy studies by Schibli et al. [43] revealed the formation of a well-defined helical structure for ${ }^{665}$ KWASLWNWFNITNWLWYIK ${ }^{683}$ peptide monomers in dodecylphosphocholine (DPC) micelles (Figure 3A, left panel). Four of the five tryptophan residues, as well as the tyrosine residue, formed a "collar" of aromatic residues along the axial length of the helix. Nuclear Overhauser effects to the headgroup and interfacial protons of protonated 
dodecylphosphocholine confirmed that the aromatic residues are positioned within the membrane-water interface of a phospholipid bilayer sustaining a "Velcro-like" interaction. The bulk of the polar residues are positioned on one face of this structure, with the hydrophobic phenylalanine side chain on the opposing face, forming an amphipathic structure.

Subsequent analyses of the MPER sequence including the application of the hydrophobic-at-interface moment to the numerous mutations generated by Salzwedel and coworkers [17] revealed that this gp41 MTR might be segmented into two segregated hydrophobic subdomains [19]. The N-terminal domain was found to span a helical amphipathic-at-interface sequence or AIS domain, a structural motif conserved among members of the lentivirinae genus [19, 91, 92]. Supporting the functional relevance of this particular organization, a number of blocking deletions invariably correlated with the ablation and/or merging of interfacial subdomains, while the effect of several Trp to Ala mutations on function correlated with the preservation of the interfacial hydrophobicity distributed into two subdomains $[19,21]$.

A more recent DPC-NMR structure reported by Sun et al. [93] provided the structural basis for the functional segmentation of this gp41 MTR (see also Figure 3A, right panel). The MPER sequence ${ }^{662}$ ELDKWASLWNWFNITNWLWYIK ${ }^{683}$ analyzed by these authors was elongated at the N-terminus by including the E662 and D664 polar residues. The conformationally defined elements present in the structure included a tilted $\alpha$-helix (664-672), a short hinge connection (673-674), and a flat helix running in parallel to the membrane plane (675-683). The N-terminal segment contained a two-turn $\alpha$-helix, whereas a one-turn $\alpha$-helix was combined with a short 310 -helix within the Cterminal segment. This kinked structure optimizes the hydrophobic surface for membrane insertion with 4 out of the 5 Trp residues plus F673 facing the 
$\mathrm{MI} /$ hydrocarbon core $(\mathrm{HC})$ interface. Moreover, side-chains of most polar residues remain solvent exposed. Remarkably, as pointed out by these authors, the membraneburied residues are the most conserved, whereas the hydrophilic-exposed residues seem to be subject of greater variability.

Low-resolution structural techniques provide additional evidence for the existence of monomeric and multimeric MPER states in a membrane environment [19]. To illustrate this point, circular dichroism (CD) spectra of MPER-derived PreTM and AISpreTM peptides are compared in Figure 3B. Spectra of monomeric helical peptides in DPC micelles display spectra with a characteristic $[\theta]_{222} /[\theta]_{208}$ ratio $<1$ (dotted traces in panel C). By comparison, CD spectra of these peptides incorporated into PC:Chol vesicles at a 1:33 peptide-to-lipid mole ratio (continuous traces) resulted in an increase of the intensity of the $222 \mathrm{~nm}$ peak. This effect was more prominent for the PreTM peptide, in which case the $[\theta]_{222} /[\theta]_{208}$ ellipticity ratio $>1$ unambiguously supports the existence of helix-helix interactions such as those described for heterodimeric coiled-coils and/or transmembrane helical bundles [94-96]. Thus, low-resolution structural data are compatible with the existence of a monomeric helical structure, prevalent in membranemimicking detergent micelles, which would self-associate at membrane surfaces. Indeed, this homo-oligomerization phenomenon is promoted by Chol and correlates with the pore-forming capacity of MPER-derived peptides [19, 21, 77].

\section{MPER as a target for fusion inhibition.}

The ability displayed by MPER-derived PreTM peptide to self-associate in membranes 
raised the possibility that formation of structurally defined MPER homo-oligomers might take place at some stage during gp41-induced fusion process [19]. It was assumed that this process might represent an alternative target for potential inhibitors. Competition with MPER self-oligomerization, and hence, fusion inhibition was tested using free PreTM peptide in HIV-1 Env-induced cell-cell fusion assays [19]. The PreTM peptide inhibited gp41-induced fusion in a dose-dependent manner with an apparent $\mathrm{IC}_{50}$ value of $577 \mathrm{nM}$. A $\operatorname{Trp}(1-3)$ Ala mutant-like peptide unable to form homo-oligomers was used as negative control and shown to be devoid of the inhibitory effect. In control experiments, neither of these two peptides inhibited influenza hemaglutinin-mediated fusion implying that PreTM inhibitory effect was through its interaction with HIV envelope protein, and not through its partitioning into cell membranes and causing lipid-mediated effects.

The potential of the lentiviral MPER region as a target for fusion inhibitor development is better exemplified in the case of the Feline Immunodeficiency Virus (FIV) transmembrane glycoprotein. A cognate 20-mer peptide derived from FIV MPER, potently inhibited viral infectivity in tissue culture $\left(\mathrm{IC}_{50} \approx 4 \mathrm{nM}\right)$ [97-99]. Antiviral activity of the leading MPER-derived sequence was found to map to a short segment containing three conserved Trp residues. Thus, further analyses focused on a derivative of eight amino acids $\left({ }^{770} \mathrm{WEDWVRWI}^{777}\right)$, designated $\mathrm{C} 8$, which still displayed potent antiviral activity $\left(\mathrm{IC}_{50} \approx 40 \mathrm{nM}\right)$. C8 activity was found to be dependent on conservation of the Trp motif, most likely because these residues are responsible for its conformational propensity. Furthermore, the retroinverso analogue of this peptide (riC8) has been shown to be effective in vivo, in experiments demonstrating its ability to decrease mean viral load when administered to chronically FIV-infected cats [99].

As mentioned in the previous section, perturbation of the integrity of lipid bilayers by 
peptide doses relevant for spike density in the viral membrane [100] and the Chol effect on this phenomenon $[101,102]$ point to membrane restructuring during fusion as the gp41 MPER functional activity. The evidence that broadly neutralizing antibodies can inhibit the membrane-restructuring processes observed in vesicular systems reinforces that idea. In particular, the broadly neutralizing anti-gp41 2F5 and 4E10 monoclonal antibodies (mAbs), which recognize linear epitopes located within MPER (reviewed in $[103,104])$, block MPER membrane activity assayed in vesicular model systems [92, 105-107]. The Fab structures solved in complex with epitope-representing peptides revealed the sequences in contact with $2 \mathrm{~F} 5\left({ }^{657}\right.$ EQELLELDKWASLW $\left.{ }^{670}\right)$ and $4 \mathrm{E} 10$ paratopes $\left({ }^{672}\right.$ WFNITNWLW $\left.{ }^{680}\right)$ [108-110]. Thus, the full 2F5 epitope roughly corresponds to the MPER AIS, while the 4E10 epitope starts at the junction between the hydrophobic subdomains and ends close to the MPER C-terminus.

These crystal structures also reveal long third complementarity-determining region of the heavy chains (CDR H3s) containing solvent-exposed aromatic residues. Accordingly, it has been suggested that 2F5 and 4E10 antibodies share a common adaptive trait for epitope recognition at the membrane surface $[108,111,112]$. Recent mutagenesis studies actually indicate that neutralizing activity might be dependent on antibody-membrane association, which would require the preservation of hydrophobicat-interface CDR H3 loop residues [113-116]. Thus, determining the optimal conditions for membrane-inserted epitope recognition and subsequent membrane activity blocking may provide useful information for the rational design of effective liposome-peptide immunogens.

In principle, antibody recognition-blocking may be conditioned by the structure and dynamics of the membrane-embedded MPER sequence, which are in turn predicted to depend on the length and composition of the MPER-derived peptide sequence 
considered (i.e., the inclusion of the different hydrophobic subdomains [117]), and the lipid composition of the model membrane (i.e., the presence of envelope lipids Chol and/or SPM [105, 106]). Figure 3 compiles results on mAb4E10-MPER peptide interactions reported in previous works $[92,105,106]$, which exemplify the effect of peptide structure on $\mathrm{mAb}$ recognition and membrane-activity blocking. The PreTM peptide is structurally represented by the interfacial helix described by Schibli et al. [43](PDB entry: 1JAV). Within this continuous structure, residues W672 and F673 face towards the $\mathrm{HC}$ region of the membrane. A comparable orientation of these residues was observed within the bipartite structure reported by Sun et al. [93] (PDB entry: 2PV6). This structure might be representative of longer MPER specimens, such as AISpreTM, bearing higher polarity at the N-terminus. Both structures also expose N671 to the solvent. In contrast, the W680 residue remains buried into the MI-HC interface within the shorter, continuous helical $1 \mathrm{JAV}$ structure, while the chain reorientation introduced by the connecting hinge induces its exposure to solvent within the longer 2PV6 structure.

Lorizate et al. [105] reported surface pressure measurements compatible with the partial extraction by 4E10 of PreTM peptide from lipid monolayers (see also Figure 4) and Sun et al. [93] provided electron-paramagnetic resonance data compatible with 4E10 epitope extraction from the longer 662-683 MPER peptide inserted into lipid bilayers. These authors observed that extracted W672 and F673 side chains rearrange within the 4E10 paratope, and that, particularly the latter residue, flips vertically and inserts deeply into the epitope-binding pocket. Sun et al. [93] also hypothesized that solvent exposed N671 and W680 were key residues in 4E10 recognition of membrane-inserted MPER by guiding the initial contacts with CDR3 regions of the antibody. Thus, given the fact that only the former would be accessible in a membrane-bound state, mAb4E10 is predicted 
to recognize-block the shorter MPER-derived PreTM peptide with lower affinity.

The Figures 3C and $\mathrm{D}$ display respectively peptide-induced leakage inhibition and antibody-vesicle association results, which, in conjunction, provide evidence for higher antibody affinity towards the membrane-bound peptide exposing W680 to solvent. 4E10 blocked the leakage process induced by the long AISpreTM peptide with an $\mathrm{IC}_{50}$ of ca. $10 \mathrm{nM}$, while an order of magnitude higher $\mathrm{IC}_{50}$ value of ca. $100 \mathrm{nM}$ was measured for the inhibition of leakage induced by the shorter PreTM peptide (Figure 3C). The actual levels of mAb-liposome association, as detected by flow-cytometry, also reflected the higher affinity of 4E10 towards vesicles containing the longer MPER peptide (Figure 3D). The implication of $\mathrm{W} 680$ in $4 \mathrm{E} 10$ recognition at the membrane surface therefore supports that this invariant residue should be exposed to the immune system in membrane-model formulations aimed at eliciting 4E10-like neutralizing antibodies.

Previously published results also support the capacity of lipids for modulating the membrane-inserted epitope recognition process. Explicitly, blocking by $4 \mathrm{E} 10$ antibody of membrane-restructuring induced by MPER-derived peptides has been shown to depend on the presence of Chol $[105,106]$, a phenomenon that suggests an adaptation of the antibody for recognition of gp41 sequences in the environment provided by the virion external membrane-interface. In contrast, 4E10 did not efficiently blocked perturbations induced in PC:SPM vesicles, presumably because the MPER epitope gets occluded in the transmembrane pores responsible for permeabilization of this type of vesicles $[105,106]$.

This point is further illustrated by the results displayed in Figure 4. The depth of insertion of peptides bound to membrane-surfaces can be assessed by determining their accessibility to proteolytic degradation by proteinase K [118]. Figure 4A displays the rhodamine fluorescence dequenching that occurs upon protease treatment of vesicles- 
containing PreTM peptide labeled with this fluorophore. Accessibility to the membranebound peptide increases in the following order $\mathrm{PC}$ :SPM $<\mathrm{PC}$ :Chol $<$ PC:SPM:Chol. Results shown in panels B and C demonstrate that the degree of accessibility correlates with the capacity of $4 \mathrm{E} 10$ for extracting the peptide from lipid monolayers and for binding to large unilamellar vesicles containing the peptide, respectively. These data also correlate the presence of Chol with the accessibility for antibody binding. Chol alters the material properties of the bilayer acting against membrane deformation [119]. However, deformation is required for generating the vacancy in the bilayer that allows MPER insertion. So, it is likely that the higher degree of phospholipid cohesion induced by Chol results in shallower insertion of the MPER epitope, which allows easier access to the antibody. In the ternary mixture, conditions for Lo-Ld fluid phase co-existence are produced [120]. We have recently shown that MPER peptides might be confined into the Ld phase in laterally segregated systems [107]. In this case, the local increase of the surface concentration of exposed peptide-epitope is likely to result in more efficient mAb binding by enhancing avidity.

In summary, the phenomenon of membrane-inserted epitope binding by anti-MPER antibodies constitutes a new paradigm of molecular recognition evolving in the particular milieu provided by the MI $[111,114,115]$. This bilayer region consists of a complex mixture of water and chemically heterogeneous phospholipid groups (polar head groups, glyceryl, phosphoryl, carbonyl and methylene groups) in which significant changes in polarity occur at short range [121]. Peptide sequence length, hydrophobicity distribution, membrane material properties and lipid composition are all parameters predicted to condition antibody recognition-blocking at the MI by modulating the conformation, aggregation state and bilayer topology adopted by the membrane-inserted MPER epitope. We surmise that upcoming effective MPER-directed immunogens will 
be built on the optimization of those parameters.

\section{ACKNOWLEDGEMENTS:}

The authors wish to thank financial support obtained from Spanish MICINN (BIO200800772) (JLN) and University of the Basque Country (GIU 06/42 and DIPE08/12) (NH and JLN). 


\section{References:}

1. Blumenthal, R.; Clague, M. J.; Durell, S. R.; Epand, R. M. Membrane fusion. Chem Rev, 2003, 103, (1), 53-69.

2. Chernomordik, L. V.; Kozlov, M. M. Protein-lipid interplay in fusion and fission of biological membranes. Annu Rev Biochem, 2003, 72, 175-207.

3. Lentz, B. R.; Malinin, V.; Haque, M. E.; Evans, K. Protein machines and lipid assemblies: current views of cell membrane fusion. Curr Opin Struct Biol, 2000, 10, (5), 607-615.

4. Zimmerberg, J.; Vogel, S. S.; Chernomordik, L. V. Mechanisms of membrane fusion. Annu Rev Biophys Biomol Struct, 1993, 22, 433-466.

5. Doms, R. W.; Moore, J. P. HIV-1 membrane fusion: targets of opportunity. $J$ Cell Biol, 2000, 151, (2), F9-14.

6. Eckert, D. M.; Kim, P. S. Mechanisms of viral membrane fusion and its inhibition. Annu Rev Biochem, 2001, 70, 777-810.

7. Gallo, S. A.; Finnegan, C. M.; Viard, M.; Raviv, Y.; Dimitrov, A.; Rawat, S. S.; Puri, A.; Durell, S.; Blumenthal, R. The HIV Env-mediated fusion reaction. Biochim Biophys Acta, 2003, 1614, (1), 36-50.

8. Wyatt, R.; Sodroski, J. The HIV-1 envelope glycoproteins: fusogens, antigens, and immunogens. Science, 1998, 280, (5371), 1884-1888.

9. Schibli, D. J.; Weissenhorn, W. Class I and class II viral fusion protein structures reveal similar principles in membrane fusion. Mol Membr Biol, 2004, 21, (6), 361-371.

10. Weissenhorn, W.; Hinz, A.; Gaudin, Y. Virus membrane fusion. FEBS Lett, 2007, 581, (11), 2150-2155.

11. Skehel, J. J.; Wiley, D. C. Receptor binding and membrane fusion in virus entry: the influenza hemagglutinin. Annu Rev Biochem, 2000, 69, 531-569.

12. Durell, S. R.; Martin, I.; Ruysschaert, J. M.; Shai, Y.; Blumenthal, R. What studies of fusion peptides tell us about viral envelope glycoprotein-mediated membrane fusion (review). Mol Membr Biol, 1997, 14, (3), 97-112.

13. Epand, R. M. Fusion peptides and the mechanism of viral fusion. Biochim Biophys Acta, 2003, 1614, (1), 116-121.

14. Nieva, J. L.; Agirre, A. Are fusion peptides a good model to study viral cell fusion? Biochim Biophys Acta, 2003, 1614, (1), 104-115.

15. Shome, S. G.; Kielian, M. Differential roles of two conserved glycine residues in the fusion peptide of Semliki Forest virus. Virology, 2001, 279, (1), 146-160.

16. Gething, M. J.; Doms, R. W.; York, D.; White, J. Studies on the mechanism of membrane fusion: site-specific mutagenesis of the hemagglutinin of influenza virus. $J$ Cell Biol, 1986, 102, (1), 11-23.

17. Salzwedel, K.; West, J. T.; Hunter, E. A conserved tryptophan-rich motif in the 
membrane-proximal region of the human immunodeficiency virus type 1 gp41 ectodomain is important for Env-mediated fusion and virus infectivity. $J$ Virol, 1999, $73,(3), 2469-2480$.

18. Suarez, T.; Gallaher, W. R.; Agirre, A.; Goni, F. M.; Nieva, J. L. Membrane interface-interacting sequences within the ectodomain of the human immunodeficiency virus type 1 envelope glycoprotein: putative role during viral fusion. $J$ Virol, 2000, 74, (17), 8038-8047.

19. Saez-Cirion, A.; Arrondo, J. L.; Gomara, M. J.; Lorizate, M.; Iloro, I.; Melikyan, G.; Nieva, J. L. Structural and functional roles of HIV-1 gp41 pretransmembrane sequence segmentation. Biophys J, 2003, 85, (6), 3769-3780.

20. Nieva, J. L.; Suarez, T. Hydrophobic-at-interface regions in viral fusion protein ectodomains. Biosci Rep, 2000, 20, (6), 519-533.

21. Lorizate, M.; Huarte, N.; Saez-Cirion, A.; Nieva, J. L. Interfacial pretransmembrane domains in viral proteins promoting membrane fusion and fission. Biochim Biophys Acta, 2008.

22. Wimley, W. C.; White, S. H. Experimentally determined hydrophobicity scale for proteins at membrane interfaces. Nat Struct Biol, 1996, 3, (10), 842-848.

23. Haffar, O. K.; Dowbenko, D. J.; Berman, P. W. Topogenic analysis of the human immunodeficiency virus type 1 envelope glycoprotein, gp160, in microsomal membranes. J Cell Biol, 1988, 107, (5), 1677-1687.

24. Suarez, T.; Nir, S.; Goni, F. M.; Saez-Cirion, A.; Nieva, J. L. The pretransmembrane region of the human immunodeficiency virus type-1 glycoprotein: a novel fusogenic sequence. FEBS Lett, 2000, 477, (1-2), 145-149.

25. Shnaper, S.; Sackett, K.; Gallo, S. A.; Blumenthal, R.; Shai, Y. The C- and the $\mathrm{N}$-terminal regions of glycoprotein 41 ectodomain fuse membranes enriched and not enriched with cholesterol, respectively. J Biol Chem, 2004, 279, (18), 18526-18534.

26. Buzon, V.; Natrajan, G.; Schibli, D.; Campelo, F.; Kozlov, M. M.; Weissenhorn, W. Crystal structure of HIV-1 gp41 including both fusion peptide and membrane proximal external regions. PLoS Pathog, 2010, 6, (5), e1000880.

27. Kliger, Y.; Shai, Y. A leucine zipper-like sequence from the cytoplasmic tail of the HIV-1 envelope glycoprotein binds and perturbs lipid bilayers. Biochemistry, 1997, $36,(17), 5157-5169$.

28. Costin, J. M.; Rausch, J. M.; Garry, R. F.; Wimley, W. C. Viroporin potential of the lentivirus lytic peptide (LLP) domains of the HIV-1 gp41 protein. Virol J, 2007, 4, 123.

29. Nieva, J. L.; Nir, S.; Muga, A.; Goni, F. M.; Wilschut, J. Interaction of the HIV1 fusion peptide with phospholipid vesicles: different structural requirements for fusion and leakage. Biochemistry, 1994, 33, (11), 3201-3209.

30. Pereira, F. B.; Goni, F. M.; Nieva, J. L. Liposome destabilization induced by the HIV-1 fusion peptide effect of a single amino acid substitution. FEBS Lett, 1995, 362, (2), 243-246.

31. Kliger, Y.; Aharoni, A.; Rapaport, D.; Jones, P.; Blumenthal, R.; Shai, Y. Fusion peptides derived from the HIV type 1 glycoprotein 41 associate within phospholipid membranes and inhibit cell-cell Fusion. Structure-function study. J Biol Chem, 1997, 
272, (21), 13496-13505.

32. Pereira, F. B.; Goni, F. M.; Muga, A.; Nieva, J. L. Permeabilization and fusion of uncharged lipid vesicles induced by the HIV-1 fusion peptide adopting an extended conformation: dose and sequence effects. Biophys J, 1997, 73, (4), 1977-1986.

33. Pereira, F. B.; Goni, F. M.; Nieva, J. L. Membrane fusion induced by the HIV type 1 fusion peptide: modulation by factors affecting glycoprotein 41 activity and potential anti-HIV compounds. AIDS Res Hum Retroviruses, 1997, 13, (14), 1203-1211.

34. Agirre, A.; Flach, C.; Goni, F. M.; Mendelsohn, R.; Valpuesta, J. M.; Wu, F.; Nieva, J. L. Interactions of the HIV-1 fusion peptide with large unilamellar vesicles and monolayers. A cryo-TEM and spectroscopic study. Biochim Biophys Acta, 2000, 1467, (1), 153-164.

35. Yang, J.; Prorok, M.; Castellino, F. J.; Weliky, D. P. Oligomeric beta structure of the membrane-bound HIV-1 fusion peptide formed from soluble monomers. Biophys. $J ., 2004,87,1951-1963$.

36. Haque, M. E.; Koppaka, V.; Axelsen, P. H.; Lentz, B. R. Properties and structures of the influenza and HIV fusion peptides on lipid membranes: implications for a role in fusion. Biophys J, 2005, 89, (5), 3183-3194.

37. Li, Y. L.; Tamm, L. K. Structure and plasticity of the human immunodeficiency virus gp41 fusion domain in lipid micelles and bilayers. Biophys. J., 2007, 93, (3), 876885 .

38. Mobley, P. W.; Waring, A. J.; Sherman, M. A.; Gordon, L. M. Membrane interactions of the synthetic N-terminal peptide of HIV-1 gp41 and its structural analogs. Biochim Biophys Acta, 1999, 1418, (1), 1-18.

39. Siegel, D. P.; Epand, R. M. The mechanism of lamellar-to-inverted hexagonal phase transitions in phosphatidylethanolamine: implications for membrane fusion mechanisms. Biophys J, 1997, 73, (6), 3089-3111.

40. Siegel, D. P.; Epand, R. M. Effect of influenza hemagglutinin fusion peptide on lamellar/inverted phase transitions in dipalmitoleoylphosphatidylethanolamine: implications for membrane fusion mechanisms. Biochim Biophys Acta, 2000, 1468, (12), 87-98.

41. Tristram-Nagle, S.; Nagle, J. F. HIV-1 fusion peptide decreases bending energy and promotes curved fusion intermediates. Biophys J, 2007, 93, (6), 2048-2055.

42. Qiang, W.; Sun, Y.; Weliky, D. P. A strong correlation between fusogenicity and membrane insertion depth of the HIV fusion peptide. Proc Natl Acad Sci U S A, 2009, 106, (36), 15314-15319.

43. Schibli, D. J.; Montelaro, R. C.; Vogel, H. J. The membrane-proximal tryptophan-rich region of the HIV glycoprotein, gp41, forms a well-defined helix in dodecylphosphocholine micelles. Biochemistry, 2001, 40, (32), 9570-9578.

44. Shnyrova, A. V.; Frolov, V. A.; Zimmerberg, J. Domain-driven morphogenesis of cellular membranes. Curr Biol, 2009, 19, (17), R772-780.

45. Kozlov, M. M. Biophysics: Joint effort bends membrane. Nature, 2010, 463, (7280), 439-440.

46. Chernomordik, L. V.; Kozlov, M. M. Mechanics of membrane fusion. Nat Struct Mol Biol, 2008, 15, (7), 675-683. 
47. Longo, M. L.; Waring, A. J.; Hammer, D. A. Interaction of the influenza hemagglutinin fusion peptide with lipid bilayers: area expansion and permeation. Biophys J, 1997, 73, (3), 1430-1439.

48. Schneider, S. E.; Bray, B. L.; Mader, C. J.; Friedrich, P. E.; Anderson, M. W.; Taylor, T. S.; Boshernitzan, N.; Niemi, T. E.; Fulcher, B. C.; Whight, S. R.; White, J. M.; Greene, R. J.; Stoltenberg, L. E.; Lichty, M. Development of HIV fusion inhibitors. J Pept Sci, 2005, 11, (11), 744-753.

49. Bosch, M. L.; Earl, P. L.; Fargnoli, K.; Picciafuoco, S.; Giombini, F.; WongStaal, F.; Franchini, G. Identification of the fusion peptide of primate immunodeficiency viruses. Science, 1989, 244, (4905), 694-697.

50. Delahunty, M. D.; Rhee, I.; Freed, E. O.; Bonifacino, J. S. Mutational analysis of the fusion peptide of the human immunodeficiency virus type 1: identification of critical glycine residues. Virology, 1996, 218, (1), 94-102.

51. Freed, E. O.; Delwart, E. L.; Buchschacher, G. L., Jr.; Panganiban, A. T. A mutation in the human immunodeficiency virus type 1 transmembrane glycoprotein gp41 dominantly interferes with fusion and infectivity. Proc Natl Acad Sci U S A, 1992, $89,(1), 70-74$.

52. Gallaher, W. R. Detection of a fusion peptide sequence in the transmembrane protein of human immunodeficiency virus. Cell, 1987, 50, (3), 327-328.

53. Hernandez, L. D.; Hoffman, L. R.; Wolfsberg, T. G.; White, J. M. Virus-cell and cell-cell fusion. Annu Rev Cell Dev Biol, 1996, 12, 627-661.

54. Longo, M. L.; Waring, A. J.; Gordon, L. M.; Hammer, D. A. Area Expansion and Permeation of Phospholipid Membrane Bilayers by Influenza Fusion Peptides and Melittin. Langmuir, 1998, 14, 2385-2395.

55. Owens, R. J.; Tanner, C. C.; Mulligan, M. J.; Srinivas, R. V.; Compans, R. W. Oligopeptide inhibitors of HIV-induced syncytium formation. AIDS Res Hum Retroviruses, 1990, 6, (11), 1289-1296.

56. Slepushkin, V. A.; Kornilaeva, G. V.; Andreev, S. M.; Sidorova, M. V.; Petrukhina, A. O.; Matsevich, G. R.; Raduk, S. V.; Grigoriev, V. B.; Makarova, T. V.; Lukashov, V. V.; et al. Inhibition of human immunodeficiency virus type 1 (HIV-1) penetration into target cells by synthetic peptides mimicking the N-terminus of the HIV1 transmembrane glycoprotein. Virology, 1993, 194, (1), 294-301.

57. Pritsker, M.; Jones, P.; Blumenthal, R.; Shai, Y. A synthetic all D-amino acid peptide corresponding to the N-terminal sequence of HIV-1 gp41 recognizes the wildtype fusion peptide in the membrane and inhibits HIV-1 envelope glycoproteinmediated cell fusion. Proc Natl Acad Sci US A, 1998, 95, (13), 7287-7292.

58. Gerber, D.; Pritsker, M.; Gunther-Ausborn, S.; Johnson, B.; Blumenthal, R.; Shai, Y. Inhibition of HIV-1 envelope glycoprotein-mediated cell fusion by a DL-amino acid-containing fusion peptide: possible recognition of the fusion complex. $J$ Biol Chem, 2004, 279, (46), 48224-48230.

59. Munch, J.; Standker, L.; Adermann, K.; Schulz, A.; Schindler, M.; Chinnadurai, R.; Pohlmann, S.; Chaipan, C.; Biet, T.; Peters, T.; Meyer, B.; Wilhelm, D.; Lu, H.; Jing, W.; Jiang, S.; Forssmann, W. G.; Kirchhoff, F. Discovery and optimization of a natural HIV-1 entry inhibitor targeting the gp41 fusion peptide. Cell, 2007, 129, (2), 263-275. 
60. Mobley, P. W.; Pilpa, R.; Brown, C.; Waring, A. J.; Gordon, L. M. Membraneperturbing domains of HIV type 1 glycoprotein 41. AIDS Res Hum Retroviruses, 2001, $17,(4), 311-327$.

61. Forssmann, W. G.; The, Y. H.; Stoll, M.; Adermann, K.; Albrecht, U.; Barlos, K.; Busmann, A.; Canales-Mayordomo, A.; Gimenez-Gallego, G.; Hirsch, J.; JimenezBarbero, J.; Meyer-Olson, D.; Munch, J.; Perez-Castells, J.; Standker, L.; Kirchhoff, F.; Schmidt, R. E. Short-Term Monotherapy in HIV-Infected Patients with a Virus Entry Inhibitor Against the gp41 Fusion Peptide. Sci Transl Med, 2010, 2, (63), 63 re63.

62. Eckert, D. M.; Malashkevich, V. N.; Hong, L. H.; Carr, P. A.; Kim, P. S. Inhibiting HIV-1 entry: discovery of D-peptide inhibitors that target the gp41 coiledcoil pocket. Cell, 1999, 99, (1), 103-115.

63. Welch, B. D.; VanDemark, A. P.; Heroux, A.; Hill, C. P.; Kay, M. S. Potent Dpeptide inhibitors of HIV-1 entry. Proc Natl Acad Sci U S A, 2007, 104, (43), 1682816833.

64. Houghten, R. A.; Appel, J. R.; Blondelle, S. E.; Cuervo, J. H.; Dooley, C. T.; Pinilla, C. The use of synthetic peptide combinatorial libraries for the identification of bioactive peptides. Biotechniques, 1992, 13, (3), 412-421.

65. Pinilla, C.; Appel, J. R.; Blanc, P.; Houghten, R. A. Rapid identification of high affinity peptide ligands using positional scanning synthetic peptide combinatorial libraries. Biotechniques, 1992, 13, (6), 901-905.

66. Lopez-Garcia, B.; Gonzalez-Candelas, L.; Perez-Paya, E.; Marcos, J. F. Identification and characterization of a hexapeptide with activity against phytopathogenic fungi that cause postharvest decay in fruits. Mol Plant Microbe Interact, 2000, 13, (8), 837-846.

67. Lopez-Garcia, B.; Perez-Paya, E.; Marcos, J. F. Identification of novel hexapeptides bioactive against phytopathogenic fungi through screening of a synthetic peptide combinatorial library. Appl Environ Microbiol, 2002, 68, (5), 2453-2460.

68. Blondelle, S. E.; Crooks, E.; Aligue, R.; Agell, N.; Bachs, O.; Esteve, V.; Tejero, R.; Celda, B.; Pastor, M. T.; Perez-Paya, E. Novel, potent calmodulin antagonists derived from an all-D hexapeptide combinatorial library that inhibit in vivo cell proliferation: activity and structural characterization. J Pept Res, 2000, 55, (2), 148-162.

69. Blondelle, S. E.; Houghten, R. A.; Perez-Paya, E. Identification of inhibitors of melittin using nonsupport-bound combinatorial libraries. J Biol Chem, 1996, 271, (8), 4093-4099.

70. Blondelle, S. E.; Houghten, R. A.; Perez-Paya, E. All D-amino acid hexapeptide inhibitors of melittin's cytolytic activity derived from synthetic combinatorial libraries. $J$ Mol Recognit, 1996, 9, (2), 163-168.

71. Ladokhin, A. S.; Wimley, W. C.; Hristova, K.; White, S. H. Mechanism of leakage of contents of membrane vesicles determined by fluorescence requenching. Methods Enzymol, 1997, 278, 474-486.

72. Nir, S.; Nieva, J. L. Interactions of peptides with liposomes: pore formation and fusion. Prog Lipid Res, 2000, 39, (2), 181-206.

73. Gomara, M. J.; Lorizate, M.; Huarte, N.; Mingarro, I.; Perez-Paya, E.; Nieva, J. L. Hexapeptides that interfere with HIV-1 fusion peptide activity in liposomes block GP41-mediated membrane fusion. FEBS Lett, 2006, 580, (11), 2561-2566. 
74. Blondelle, S. E.; Perez-Paya, E.; Houghten, R. A. Synthetic combinatorial libraries: novel discovery strategy for identification of antimicrobial agents. Antimicrob Agents Chemother, 1996, 40, (5), 1067-1071.

75. Munoz-Barroso, I.; Salzwedel, K.; Hunter, E.; Blumenthal, R. Role of the membrane-proximal domain in the initial stages of human immunodeficiency virus type 1 envelope glycoprotein-mediated membrane fusion. $J$ Virol, 1999, 73, (7), 6089-6092.

76. Kliger, Y.; Gallo, S. A.; Peisajovich, S. G.; Munoz-Barroso, I.; Avkin, S.; Blumenthal, R.; Shai, Y. Mode of action of an antiviral peptide from HIV-1. Inhibition at a post-lipid mixing stage. J Biol Chem, 2001, 276, (2), 1391-1397.

77. Saez-Cirion, A.; Nir, S.; Lorizate, M.; Agirre, A.; Cruz, A.; Perez-Gil, J.; Nieva, J. L. Sphingomyelin and cholesterol promote HIV-1 gp41 pretransmembrane sequence surface aggregation and membrane restructuring. J Biol Chem, 2002, 277, (24), 2177621785 .

78. Aloia, R. C.; Jensen, F. C.; Curtain, C. C.; Mobley, P. W.; Gordon, L. M. Lipid composition and fluidity of the human immunodeficiency virus. Proc Natl Acad Sci US $A$, 1988, 85, (3), 900-904.

79. Brügger, B.; Glass, B.; Haberkant, P.; Leibrecht, I.; Wieland, F. T.; Kräusslich, H. G. The HIV lipidome: a raft with an unusual composition. Proc Natl Acad Sci U S A, 2006, 103, (8), 2641-2646.

80. Lorizate, M.; Brügger, B.; Akiyama, H.; Glass, B.; Muller, B.; Anderluh, G.; Wieland, F. T.; Kräusslich, H. G. Probing HIV-1 membrane liquid order by Laurdan staining reveals producer cell-dependent differences. J Biol Chem, 2009, 284, (33), 22238-22247.

81. Nguyen, D. H.; Hildreth, J. E. Evidence for budding of human immunodeficiency virus type 1 selectively from glycolipid-enriched membrane lipid rafts. J Virol, 2000, 74, (7), 3264-3272.

82. Campbell, S. M.; Crowe, S. M.; Mak, J. Virion-associated cholesterol is critical for the maintenance of HIV-1 structure and infectivity. Aids, 2002, 16, (17), 2253-2261.

83. Vincent, N.; Genin, C.; Malvoisin, E. Identification of a conserved domain of the HIV-1 transmembrane protein gp41 which interacts with cholesteryl groups. Biochim Biophys Acta, 2002, 1567, (1-2), 157-164.

84. Epand, R. F.; Sayer, B. G.; Epand, R. M. The tryptophan-rich region of HIV gp41 and the promotion of cholesterol-rich domains. Biochemistry, 2005, 44, (14), $5525-5531$.

85. Vishwanathan, S. A.; Thomas, A.; Brasseur, R.; Epand, R. F.; Hunter, E.; Epand, R. M. Hydrophobic substitutions in the first residue of the CRAC segment of the gp41 protein of HIV. Biochemistry, 2008, 47, (1), 124-130.

86. Chen, S. S.; Yang, P.; Ke, P. Y.; Li, H. F.; Chan, W. E.; Chang, D. K.; Chuang, C. K.; Tsai, Y.; Huang, S. C. Identification of the LWYIK motif located in the human immunodeficiency virus type 1 transmembrane gp41 protein as a distinct determinant for viral infection. $J$ Virol, 2009, 83, (2), 870-883.

87. Schroeder, C. Cholesterol-binding viral proteins in virus entry and morphogenesis. Subcell Biochem, 2010, 51, 77-108.

88. Epand, R. F.; Thomas, A.; Brasseur, R.; Vishwanathan, S. A.; Hunter, E.; Epand, 
R. M. Juxtamembrane protein segments that contribute to recruitment of cholesterol into domains. Biochemistry, 2006, 45, (19), 6105-6114.

89. Vishwanathan, S. A.; Thomas, A.; Brasseur, R.; Epand, R. F.; Hunter, E.; Epand, R. M. Large changes in the CRAC segment of gp41 of HIV do not destroy fusion activity if the segment interacts with cholesterol. Biochemistry, 2008, 47, (45), 1186911876.

90. Vishwanathan, S. A.; Hunter, E. Importance of the membrane-perturbing properties of the membrane-proximal external region of human immunodeficiency virus type $1 \mathrm{gp} 41$ to viral fusion. J Virol, 2008, 82, (11), 5118-5126.

91. Lorizate, M.; de la Arada, I.; Huarte, N.; Sanchez-Martinez, S.; de la Torre, B. G.; Andreu, D.; Arrondo, J. L.; Nieva, J. L. Structural analysis and assembly of the HIV-1 Gp41 amino-terminal fusion peptide and the pretransmembrane amphipathic-atinterface sequence. Biochemistry, 2006, 45, (48), 14337-14346.

92. Huarte, N.; Lorizate, M.; Maeso, R.; Kunert, R.; Arranz, R.; Valpuesta, J. M.; Nieva, J. L. The broadly neutralizing anti-human immunodeficiency virus type 1 4E10 monoclonal antibody is better adapted to membrane-bound epitope recognition and blocking than 2F5. J Virol, 2008, 82, (18), 8986-8996.

93. Sun, Z. Y.; Oh, K. J.; Kim, M.; Yu, J.; Brusic, V.; Song, L.; Qiao, Z.; Wang, J. H.; Wagner, G.; Reinherz, E. L. HIV-1 broadly neutralizing antibody extracts its epitope from a kinked gp41 ectodomain region on the viral membrane. Immunity, 2008, $28,(1), 52-63$.

94. Keating, A. E.; Malashkevich, V. N.; Tidor, B.; Kim, P. S. Side-chain repacking calculations for predicting structures and stabilities of heterodimeric coiled coils. Proc Natl Acad Sci US A, 2001, 98, (26), 14825-14830.

95. Litowski, J. R.; Hodges, R. S. Designing heterodimeric two-stranded alphahelical coiled-coils. Effects of hydrophobicity and alpha-helical propensity on protein folding, stability, and specificity. J Biol Chem, 2002, 277, (40), 37272-37279.

96. Park, K.; Perczel, A.; Fasman, G. D. Differentiation between transmembrane helices and peripheral helices by the deconvolution of circular dichroism spectra of membrane proteins. Protein Sci, 1992, 1, (8), 1032-1049.

97. Lombardi, S.; Massi, C.; Indino, E.; La Rosa, C.; Mazzetti, P.; Falcone, M. L.; Rovero, P.; Fissi, A.; Pieroni, O.; Bandecchi, P.; Esposito, F.; Tozzini, F.; Bendinelli, M.; Garzelli, C. Inhibition of feline immunodeficiency virus infection in vitro by envelope glycoprotein synthetic peptides. Virology, 1996, 220, (2), 274-284.

98. Giannecchini, S.; Di Fenza, A.; D'Ursi, A. M.; Matteucci, D.; Rovero, P.; Bendinelli, M. Antiviral activity and conformational features of an octapeptide derived from the membrane-proximal ectodomain of the feline immunodeficiency virus transmembrane glycoprotein. J Virol, 2003, 77, (6), 3724-3733.

99. Giannecchini, S.; Alcaro, M. C.; Isola, P.; Sichi, O.; Pistello, M.; Papini, A. M.; Rovero, P.; Bendinelli, M. Feline immunodeficiency virus plasma load reduction by a retroinverso octapeptide reproducing the Trp-rich motif of the transmembrane glycoprotein. Antivir Ther, 2005, 10, (5), 671-680.

100. Roux, K. H.; Taylor, K. A. AIDS virus envelope spike structure. Curr Opin Struct Biol, 2007, 17, (2), 244-252.

101. Campbell, S.; Gaus, K.; Bittman, R.; Jessup, W.; Crowe, S.; Mak, J. The raft- 
promoting property of virion-associated cholesterol, but not the presence of virionassociated Brij 98 rafts, is a determinant of human immunodeficiency virus type 1 infectivity. $J$ Virol, 2004, 78, (19), 10556-10565.

102. Liao, Z.; Graham, D. R.; Hildreth, J. E. Lipid rafts and HIV pathogenesis: virion-associated cholesterol is required for fusion and infection of susceptible cells. AIDS Res Hum Retroviruses, 2003, 19, (8), 675-687.

103. Zwick, M. B. The membrane-proximal external region of HIV-1 gp41: a vaccine target worth exploring. Aids, 2005, 19, (16), 1725-1737.

104. Montero, M.; van Houten, N. E.; Wang, X.; Scott, J. K. The membrane-proximal external region of the human immunodeficiency virus type 1 envelope: dominant site of antibody neutralization and target for vaccine design. Microbiol Mol Biol Rev, 2008, 72, (1), 54-84, table of contents.

105. Lorizate, M.; Cruz, A.; Huarte, N.; Kunert, R.; Perez-Gil, J.; Nieva, J. L. Recognition and blocking of HIV-1 gp41 pre-transmembrane sequence by monoclonal 4E10 antibody in a Raft-like membrane environment. J Biol Chem, 2006, 281, (51), 39598-39606.

106. Huarte, N.; Lorizate, M.; Kunert, R.; Nieva, J. L. Lipid modulation of membrane-bound epitope recognition and blocking by HIV-1 neutralizing antibodies. FEBS Lett, 2008, 582, (27), 3798-3804.

107. Apellaniz, B.; Garcia-Saez, A. J.; Huarte, N.; Kunert, R.; Vorauer-Uhl, K.; Katinger, H.; Schwille, P.; Nieva, J. L. Confocal microscopy of giant vesicles supports the absence of HIV-1 neutralizing 2F5 antibody reactivity to plasma membrane phospholipids. FEBS Lett, 2010, 584, (8), 1591-1596.

108. Ofek, G.; Tang, M.; Sambor, A.; Katinger, H.; Mascola, J. R.; Wyatt, R.; Kwong, P. D. Structure and mechanistic analysis of the anti-human immunodeficiency virus type 1 antibody 2F5 in complex with its gp41 epitope. J Virol, 2004, 78, (19), 10724-10737.

109. Cardoso, R. M.; Brunel, F. M.; Ferguson, S.; Zwick, M.; Burton, D. R.; Dawson, P. E.; Wilson, I. A. Structural basis of enhanced binding of extended and helically constrained peptide epitopes of the broadly neutralizing HIV-1 antibody 4E10. $J \mathrm{Mol}$ Biol, 2007, 365, (5), 1533-1544.

110. Julien, J. P.; Bryson, S.; Nieva, J. L.; Pai, E. F. Structural Details of HIV-1 Recognition by the Broadly Neutralizing Monoclonal Antibody 2F5: Epitope Conformation, Antigen-Recognition Loop Mobility, and Anion-Binding Site. $J$ Mol Biol, 2008, 384, 377-392.

111. Sanchez-Martinez, S.; Lorizate, M.; Katinger, H.; Kunert, R.; Nieva, J. L. Membrane association and epitope recognition by HIV-1 neutralizing anti-gp41 2F5 and 4E10 antibodies. AIDS Res Hum Retroviruses, 2006, 22, (10), 998-1006.

112. Song, L.; Sun, Z. Y.; Coleman, K. E.; Zwick, M. B.; Gach, J. S.; Wang, J. H.; Reinherz, E. L.; Wagner, G.; Kim, M. Broadly neutralizing anti-HIV-1 antibodies disrupt a hinge-related function of gp41 at the membrane interface. Proc Natl Acad Sci $U S A$, 2009, 106, (22), 9057-9062.

113. Alam, S. M.; Morelli, M.; Dennison, S. M.; Liao, H. X.; Zhang, R.; Xia, S. M.; Rits-Volloch, S.; Sun, L.; Harrison, S. C.; Haynes, B. F.; Chen, B. Role of HIV membrane in neutralization by two broadly neutralizing antibodies. Proc Natl Acad Sci 
US A, 2009, 106, (48), 20234-20239.

114. Ofek, G.; McKee, K.; Yang, Y.; Yang, Z. Y.; Skinner, J.; Guenaga, F. J.; Wyatt, R.; Zwick, M. B.; Nabel, G. J.; Mascola, J. R.; Kwong, P. D. Relationship between antibody 2F5 neutralization of HIV-1 and hydrophobicity of its heavy chain third complementarity-determining region. $J$ Virol, 2010, 84, (6), 2955-2962.

115. Scherer, E. M.; Leaman, D. P.; Zwick, M. B.; McMichael, A. J.; Burton, D. R. Aromatic residues at the edge of the antibody combining site facilitate viral glycoprotein recognition through membrane interactions. Proc Natl Acad Sci U S A, 2010, 107, (4), 1529-1534.

116. Julien, J. P.; Huarte, N.; Maeso, R.; Taneva, S. G.; Cunningham, A.; Nieva, J. L.; Pai, E. F. Ablation of the complementarity-determining region H3 apex of the antiHIV-1 broadly neutralizing antibody 2F5 abrogates neutralizing capacity without affecting core epitope binding. J Virol, 2010, 84, (9), 4136-4147.

117. Apellaniz, B.; Nir, S.; Nieva, J. L. Distinct mechanisms of lipid bilayer perturbation induced by peptides derived from the membrane-proximal external region of HIV-1 gp41. Biochemistry, 2009, 48, (23), 5320-5331.

118. Oren, Z.; Lerman, J. C.; Gudmundsson, G. H.; Agerberth, B.; Shai, Y. Structure and organization of the human antimicrobial peptide LL-37 in phospholipid membranes: relevance to the molecular basis for its non-cell-selective activity. Biochem J, 1999, 341 ( Pt 3), 501-513.

119. McIntosh, T. J.; Simon, S. A. Bilayers as protein solvents: role of bilayer structure and elastic properties. J Gen Physiol, 2007, 130, (2), 225-227.

120. Lingwood, D.; Simons, K. Lipid rafts as a membrane-organizing principle. Science, 2010, 327, (5961), 46-50.

121. White, S. H.; Wimley, W. C. Membrane protein folding and stability: physical principles. Annu Rev Biophys Biomol Struct, 1999, 28, 319-365. 


\section{Figure Legends:}

Figure1: Membrane-transferring regions (MTRs) of gp41 ectodomain. A) Diagram showing the potential to transfer from water into membrane interfaces of the sequences located at both ends of the gp41 ectodomain helical hairpin (for clarity, only one monomer of the trimeric bundle is shown). The average hydropathy plots represent mean values for a window of 11 amino acids, calculated according to the Wimley-White hydrophobicity-at-interface scale [22]. Designated regions include: FP, fusion peptide; NHR and CHR, amino- and carboxy-terminal helical regions, respectively; MPER, membrane-proximal external region; TMD, transmembrane domain. B) Model for MTR activity during gp41-induced membrane fusion. Gp120 subunit, which is likely to shelter most of the ectodomain surface, has been omitted for the sake of clarity. I) In the gp41 native structure MTRs fold as part of the globular ectodomain. Positions as drawn in the model are not based on experimental evidence, since the native ectodomain's structure has not been solved with atomic resolution, and the actual organization of the MTRs within remains unknown. II) Pre-hairpin intermediate. Upon activation of the fusion cascade, MTRs are set free for insertion into the cell and viral membranes. Insertion restricted to one leaflet of the lipid bilayer is proposed to generate curved lipidic structures that prime the membrane for fusion. Even though the model suggests that the complete MTR-s submerge inside the membrane, insertion may involve only part of these regions. III) Formation of the hairpin. Membrane fusion evolves coupled to the completion of the 6-HB. It is assumed, but not experimentally proven, that MTRs and TMD-s may form membrane-bound complexes. 
Figure 2: Isolation of fusion inhibitors based on their capacity for interfering with FP pore-forming activity. A) Mechanistic model for pore formation in vesicles by membrane active MTR peptides [72]. The model assumes that: (1) the peptides bind and become incorporated into the bilayer of the vesicles almost instantaneously; (2) coupled to partitioning the peptides adopt a defined secondary structure (an $\alpha$-helix in this example); (3) peptide aggregation and translocation occurs within membranes; and (4) when an aggregate has reached a critical size, a channel or pore can be created within the membrane, and leakage of encapsulated molecules can occur. B) Screening of all Damino acid $\mathrm{Ac}-\mathrm{xxO}_{3} \mathrm{xxx}-\mathrm{NH} 2$ hexapeptide sub-library for inhibition of FP pore-forming activity. The panel represents a set of 19 hexapeptide mixtures having a defined residue at position 3 (X axis). The bars represent percentages of leakage increase (i.e., negative increase reflects inhibition). Means of four independent measurements are plotted. C) Effects exerted by the SCL-derived inhibitory all D-amino acid hexapeptides Acgqidev-NH2 (82-2) and Ac-gqidqv-NH2 (82-5) on FP-induced POPG LUV permeabilization. In these assays the FP concentration was $4 \mu \mathrm{M}$ and the hexapeptides were added at the FP-to-hexapeptide mole ratio of 1:40 (addition time indicated by the arrow). The dotted line corresponds to the fluorescence intensity increase that follows NBD-labeled FP insertion into the membrane. The inset further shows that the NBD signal had leveled off by the time the inhibitors were added. D) Dose dependency of FP-induced leakage inhibition by $82-2$ and $82-5$ (circles and squares, respectively). Dhexapetides were either pre-incubated in DMSO with FP (dotted lines and filled symbols) or added to the ongoing leakage process as in the previous panel (continuous lines and hollow symbols). E) Effect of 82-2 and 82-5 on syncytium formation induced by expressed gp120/41. Top: Co-incubation of the effector and target cells at $37{ }^{\circ} \mathrm{C}$ in the absence of peptides resulted in extensive fusion, as evidenced by the formation of 
syncytia (CTL panel). When hexapeptides were present in the external solution, they inhibited fusion as evidenced by the large decrease in multinucleated cells in the culture (+82-2 panel). Bottom: Quantitation of cell-cell fusion inhibition by 82-2 (hollow symbols and continuous line) and 82-5 (filled symbols and dashed line). Means of two independent measurements are displayed. Lines correspond to best fits to hyperbolic functions.

Figure 3: Sequence context effect on 4 E10 antibody recognition-blocking of MPER epitope immersed in membranes. A) Comparison of NMR structures of ${ }^{665}$ KWASLWNWFNITNWLWYIK $^{683}$ and ${ }^{662}$ ELDKWASLWNWFNITNWLWYIK ${ }^{683}$ peptides in DPC micelles (structures, derived from 1JAV [43] and 2PV6 [93] PDB entries, respectively, were rendered with the Swiss-PDB-viewer program). Helices are oriented with side chains facing the membrane $\mathrm{HC}$ region projecting downwards. 4E10 epitope spans the dark gray stretch. B) CD spectra of PreTM $\left({ }^{664}\right.$ DKWASLWNWFNITNWLWYIK ${ }^{683}$, left panel) and AISpreTM $\left({ }^{656}\right.$ NEQELLELDKWASLWNWFNITNWLWYIK ${ }^{683}$, right panel) in DPC (5 mM) micelles (dotted) and POPC:Chol vesicles (continuous trace). Lipid-alone signal was subtracted from these samples. C) mAb4E10 inhibition of ANTS leakage induced by PreTM and AISpreTM (left and right panels, respectively). The antibody was added to vesicles 20 seconds after the peptide [92, 105]. Inhibition percentages of ongoing leakage were plotted as a function of mAb4E10 concentration. The dotted lines correspond to best fits to hyperbolic functions. D) $4 \mathrm{E} 10 \mathrm{mAb}$ association with POPC:Chol large unilamellar vesicles containing PreTM (left panel) or AISpreTM (right panel) as determined by flow cytometry [92]. To match conditions in the previous panel, vesicles were pre-incubated for 20 seconds with peptide at a 1:100 peptide-to- 
lipid molar ratio, and subsequently incubated for 10 minutes with 0 (dot line), 10 (slash line), 25 (slash dot line) or 40 (continuous line) $\mu \mathrm{g} / \mathrm{ml}$ of mAb4E10.

Figure 4: Lipid composition effect on 4 E10 antibody recognition-blocking of MPER epitope immersed in membranes. A) Accessibility of membrane-bound peptides to proteolytic degradation. Vesicles (100 $\mu \mathrm{M}$ lipid) containing Rhodaminelabelled PreTM peptide (1:500 peptide to lipid mole ratio) were treated with Proteinase $\mathrm{K}(60 \mu \mathrm{g} / \mathrm{ml}$, addition time indicated by the arrow). The kinetic traces follow the rhodamine fluorescence increase as a function of time. B) mAb4E10 recognitionextraction of PreTM sequence inserted into lipid monolayers. The panels show the decrease in lateral surface pressure of peptide-containing monolayers induced upon injection of $4 \mathrm{E} 10$ antibody into the subphases $(25 \mu \mathrm{g}$, at the time indicated by the arrow). Conditions otherwise as those described in reference [105]. C) 4E10 mAb association with PreTM-containing vesicles as determined by flow cytometry [92]. Vesicles $(0.25 \mathrm{mM}$ lipid $)$ pre-incubated for 5 minutes with peptide at a 1:100 peptideto-lipid molar ratio, were subsequently incubated for 10 minutes with 0 (dot line), 5 (slash line) or 20 (continuous line) $\mu \mathrm{g} / \mathrm{ml}$ of mAb4E10. 
512AVGIGALFLGFLGAAGSTMGARS534

A

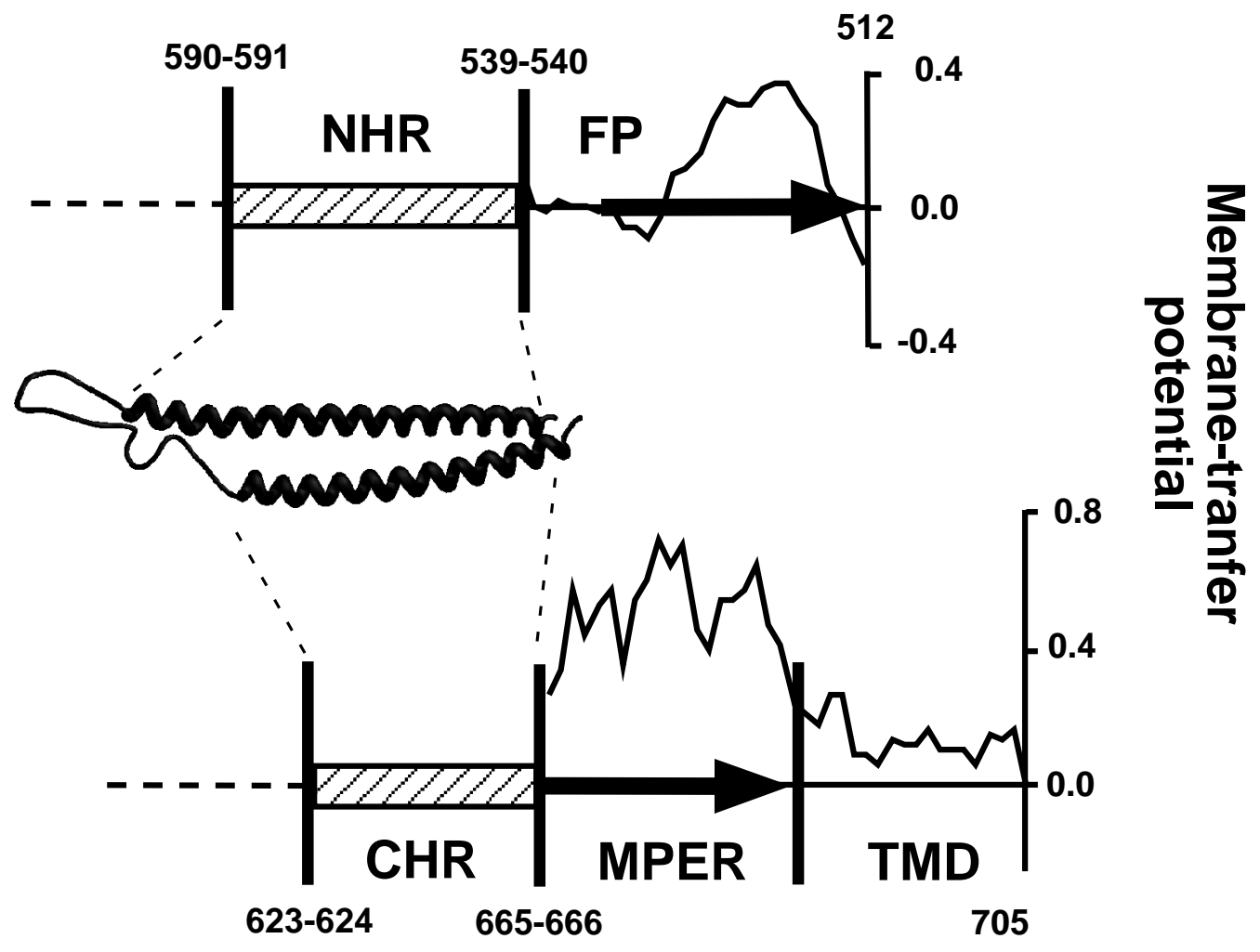

664DKWASLWNWFNITNWLWYIK683

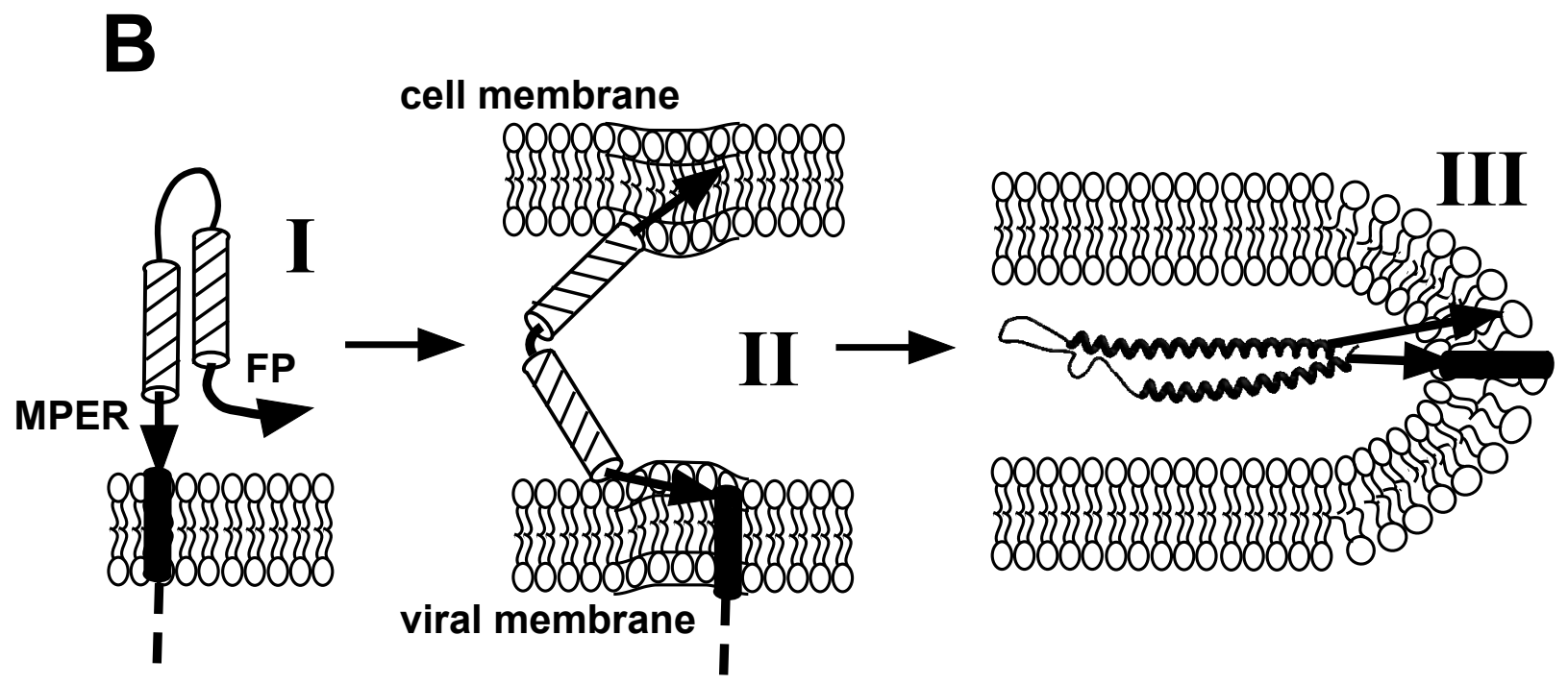




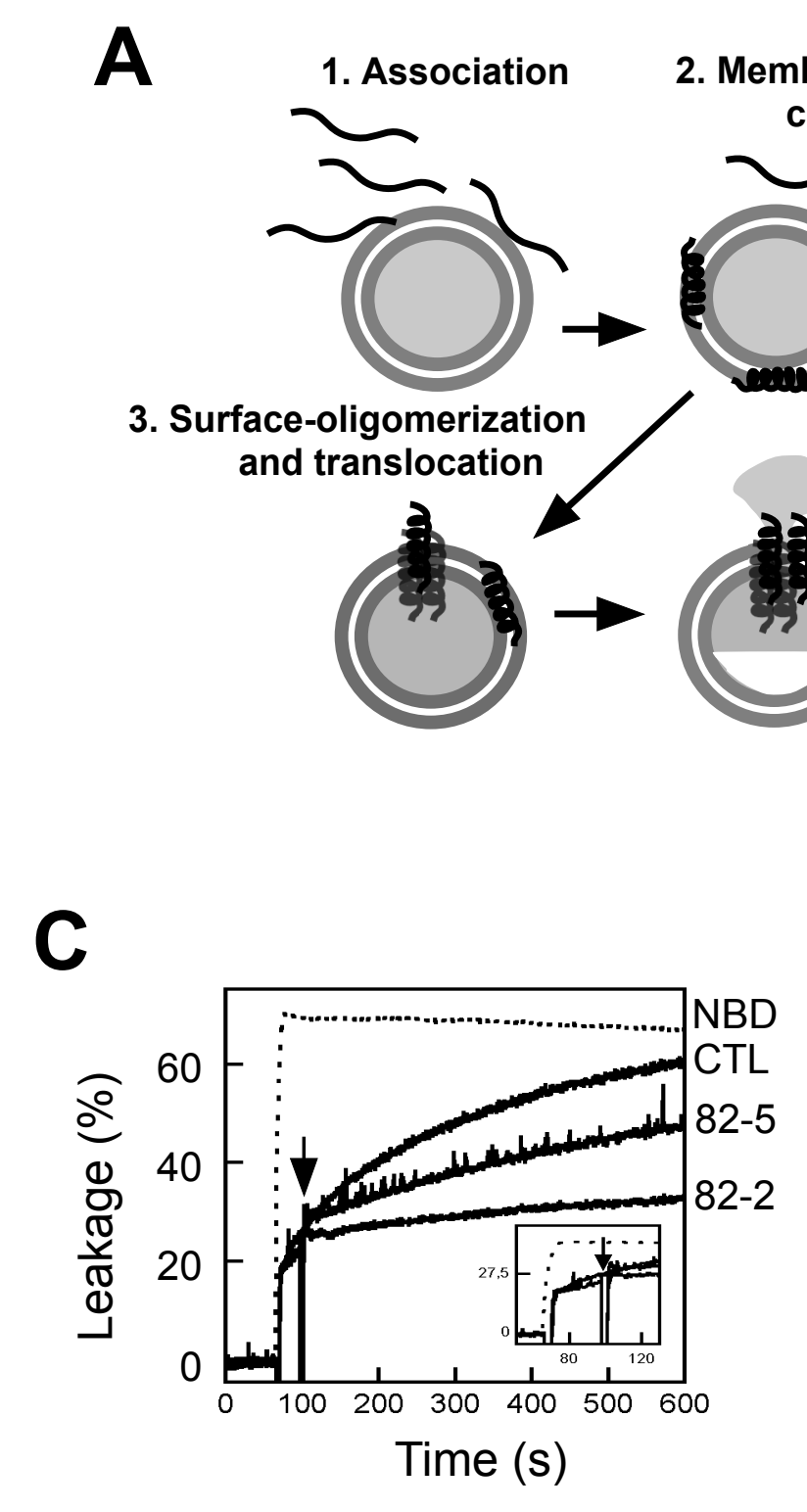

4. Pore-opening

brane-partitioning
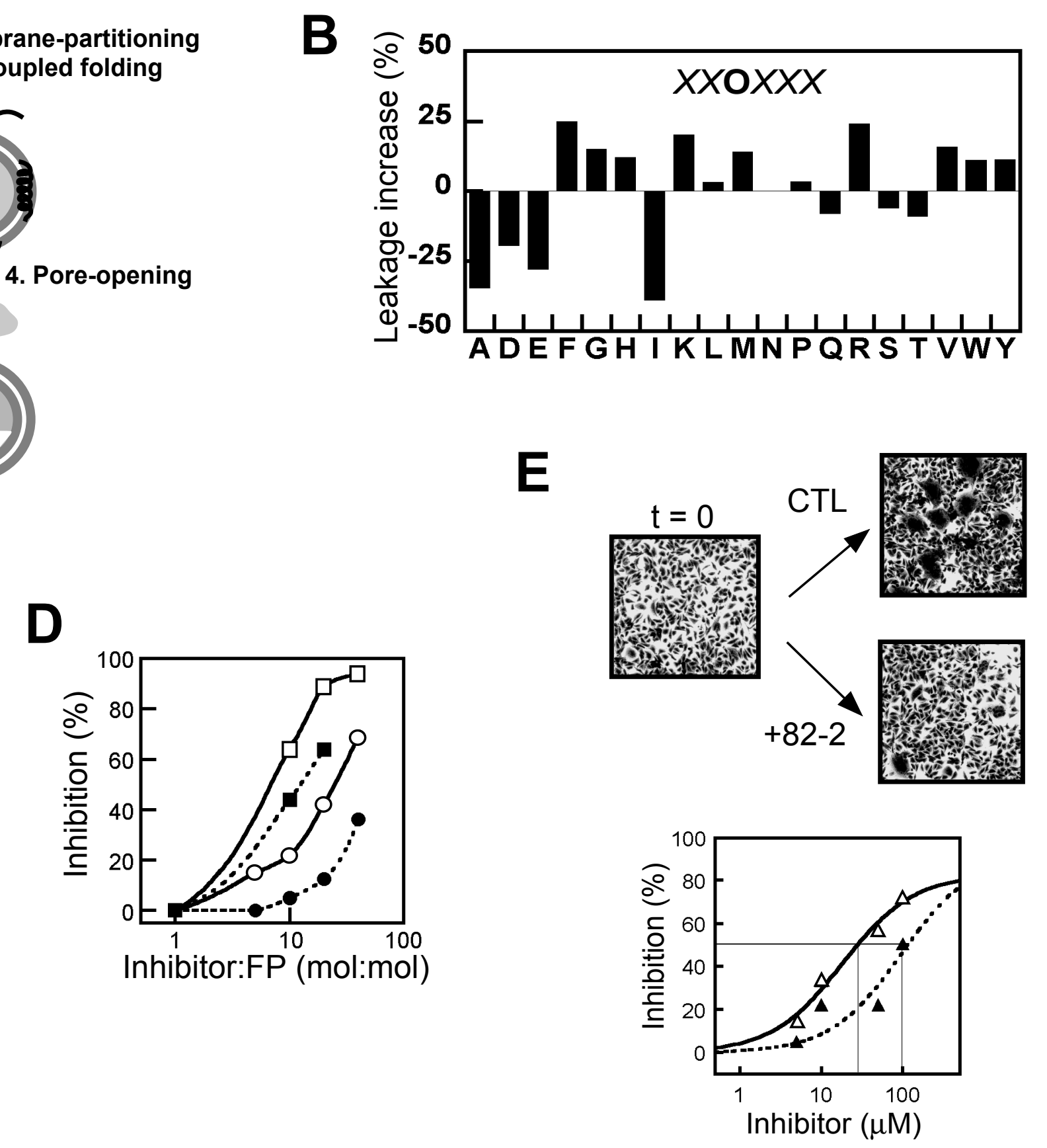
A
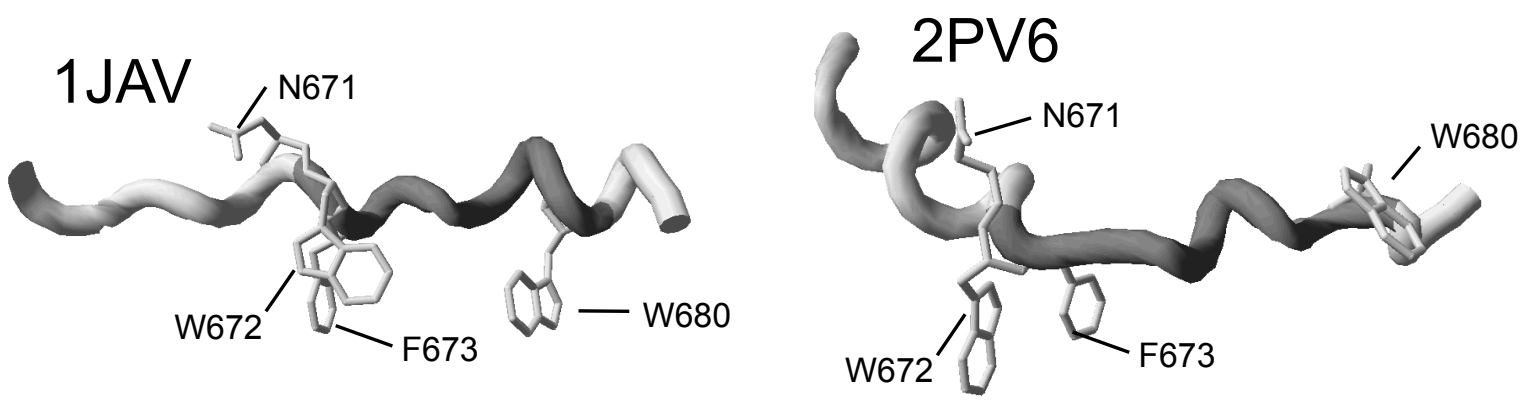

B

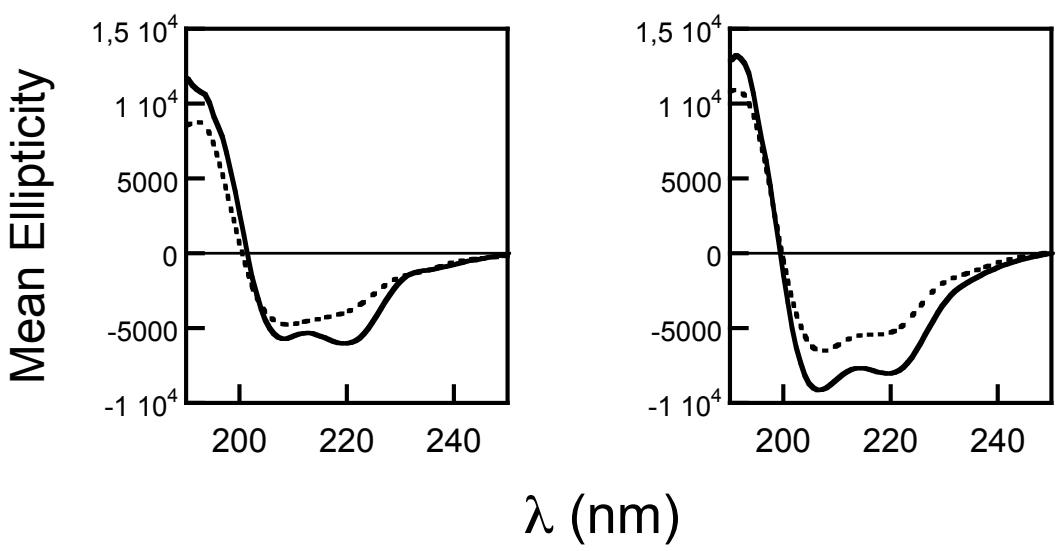

C

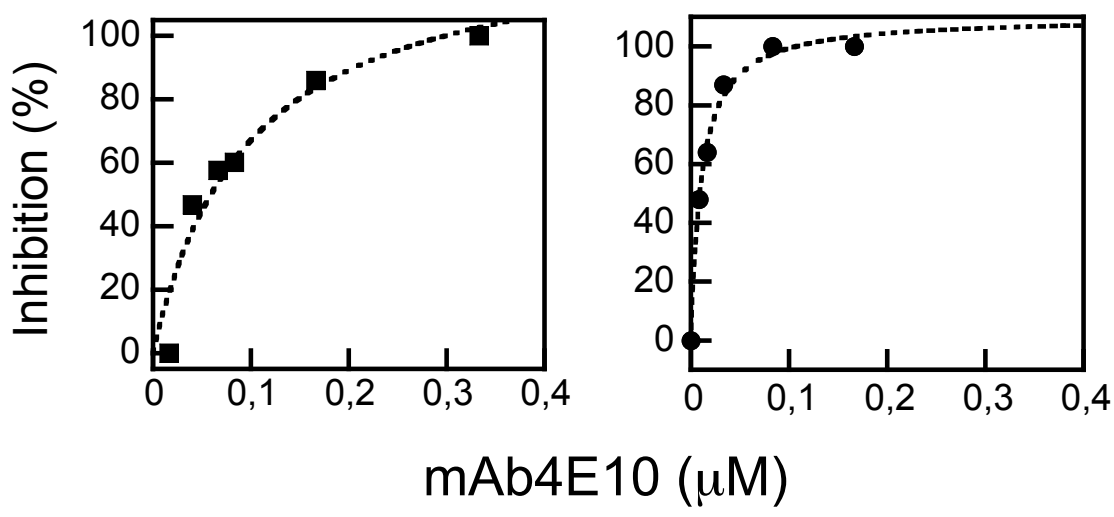

D
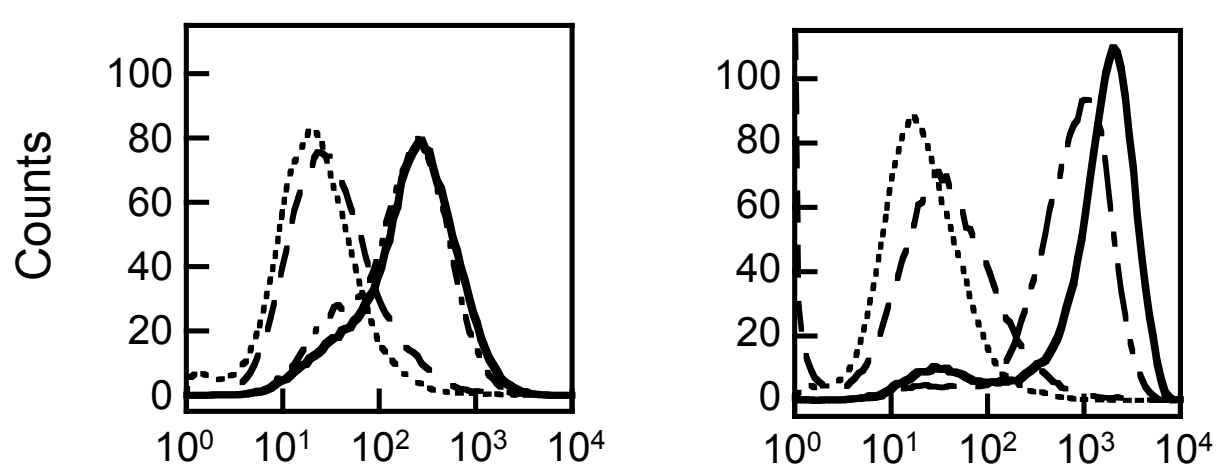

Fluorescence 
A

PC:SPM
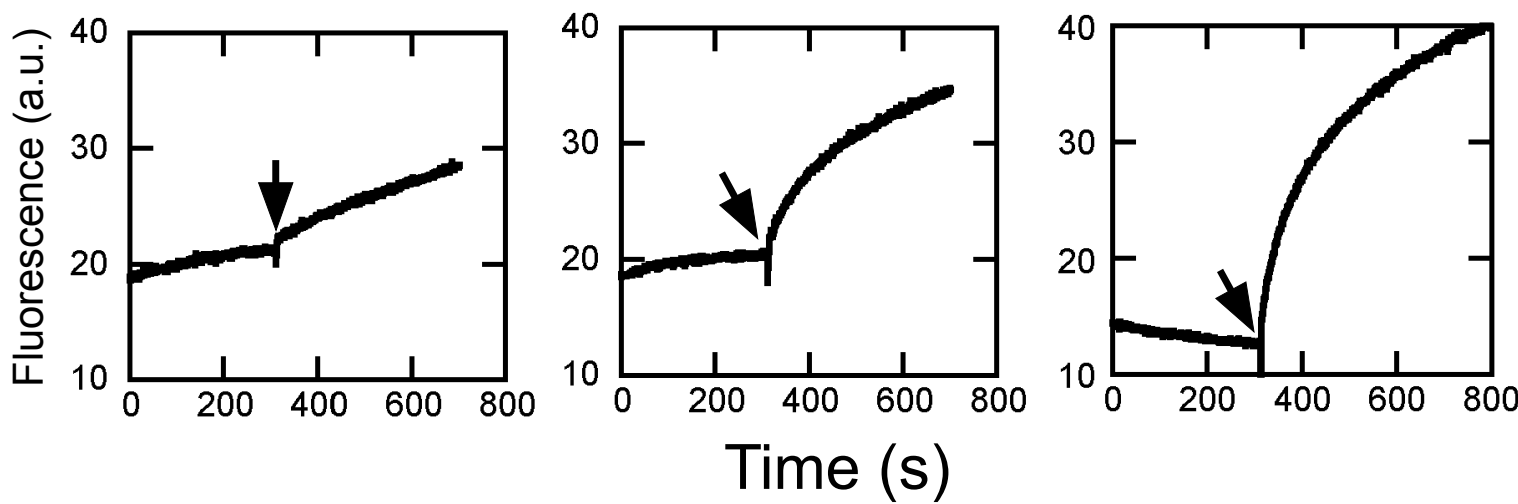

B
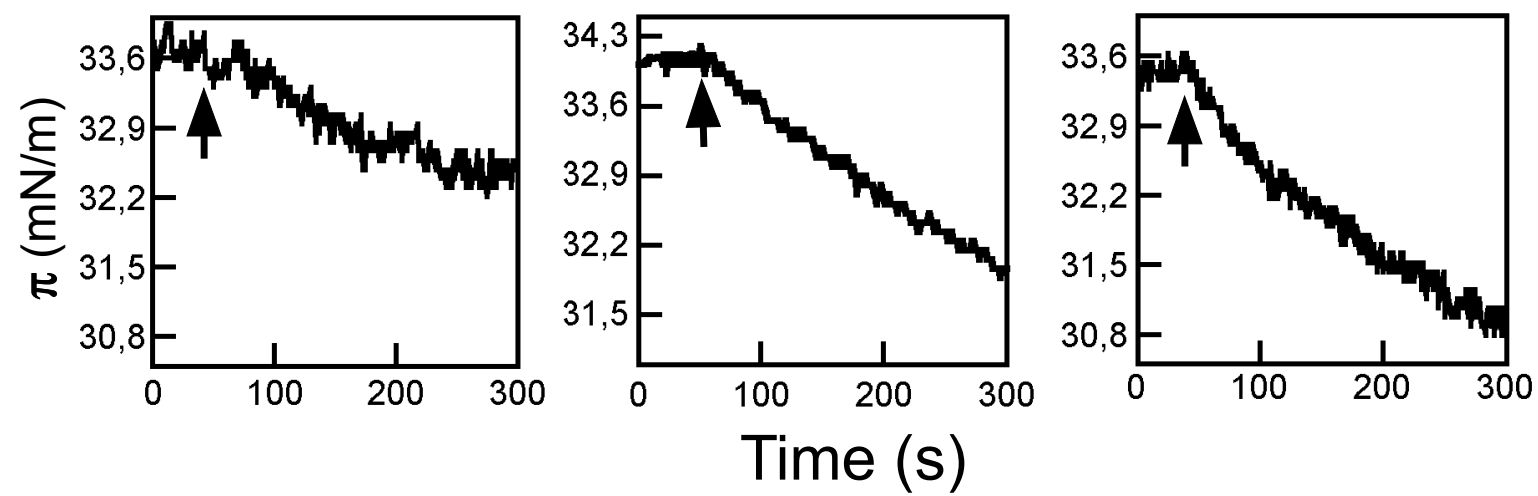

C
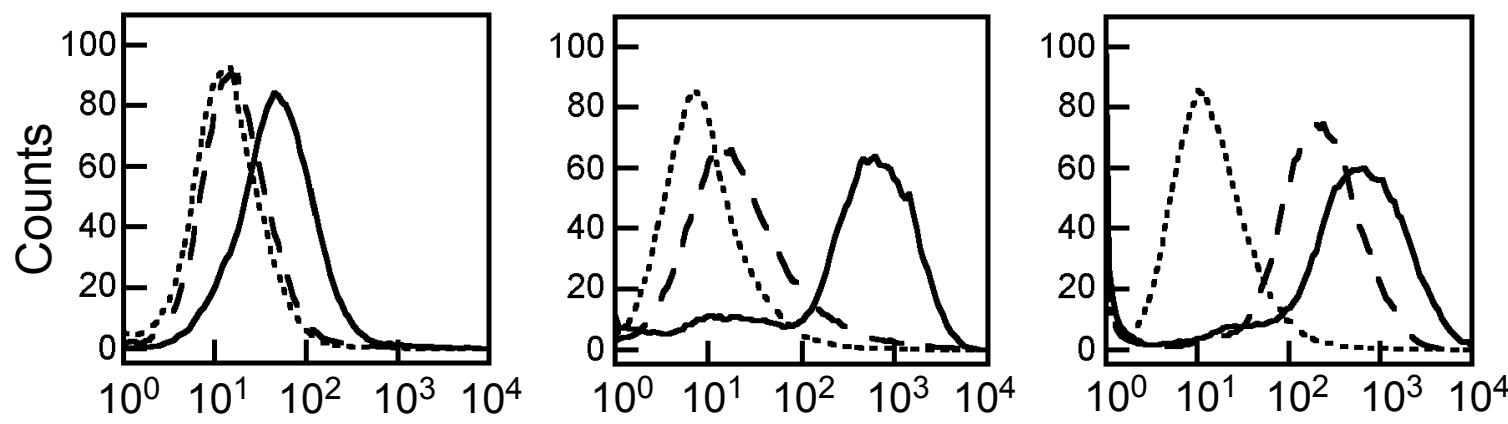

Fluorescence 